\title{
LORENZO ZAMBONI
}

\section{Ceramiche d'impasto decorate in Cisalpina tra seconda età del Ferro e romanizzazione - appunti per una ricerca}

\begin{abstract}
Come già intuito da Maria Teresa Grassi, le classi ceramiche domestiche decorate sono un aspetto sottovalutato ma cruciale per decifrare la complessità delle interazioni culturali nelle regioni della Cisalpina centrale (Piemonte orientale, Lombardia, Emilia e Veneto occidentale). Il periodo considerato è compreso tra la metà del I millennio a.C. e la prima età imperiale, quando la pianura padana è un mosaico di culture e attori sociali in movimento che lasciano tracce nella cultura materiale sfuggenti alle categorie interpretative tradizionali. In questo lavoro si pongono le basi - tecniche e teoriche - per una riconsiderazione di quelle ceramiche d'impasto, realizzate a mano o al tornio lento, decorate con tecniche che traggono ispirazione da diverse regioni e culture (Liguria, Europa centrale), andando a formare un aspetto culturale che caratterizza abitati e necropoli dell'Italia settentrionale.
\end{abstract}

Parole chiave - Pianura padana; età del Ferro; romanizzazione; ceramiche decorate; interazione culturale

Title - Decorated coarse ware in Northern Italy between the Late Iron Age and the romanization process. Research notes.

Abstract - As Maria Teresa Grassi already highlighted, decorated coarse ware represents an underestimated and yet a key aspect for deciphering the complexity of cultural interactions in central Cisalpine regions (i.e., eastern Piedmont, Lombardy, Emilia, and western Veneto). The period addressed is the second half of the $1^{\text {st }}$ millennium $\mathrm{BC}$ to the early imperial age when the Po valley is a mosaic of cultures and social actors on the move, and whose material culture challenges traditional interpretative categories. The aim of this paper is to discuss some technical and theoretical issues related to a specific class of coarse ware. This pottery, handmade or manufactured on the slow potter's wheel, is decorated with styles and techniques influenced by non-local neighbouring cultures (e.g., Liguria, Central Europe), showing a super-regional and hybridized cultural aspect.

Keywords - Po valley; Iron Age; Romanization; Decorated coarse ware; Cultural interaction

\section{Introduzione}

«Alcuni materiali documentano però una certa resistenza della tradizione indigena alla sempre più invadente presenza di manufatti romani [...]. È piuttosto interessante, quindi, che la persistenza di caratteristiche culturali indigene non sia affidata soltanto, come accade frequentemente, a oggetti pertinenti all'abbigliamento e all'ornamento, ma si riveli anche attraverso alcuni manufatti fittili [...] ollette e ciotole caratterizzate da una esuberante decorazione [...]. Ed è proprio la ricchezza dei motivi decorativi, variamente combinati, l'elemento distintivo e di maggior interesse di questa produzione» (GRASSI 2000, p. 19)

«Tumultuous times breed tumultuous material culture» (COBB - DEPRATTER 2012, p. 456)

Uno dei contributi di Maria Teresa Grassi - e uno dei suoi primi e più vivi interessi - riguarda le ricerche in territorio lombardo tra il tardo La Tène e la piena romanità, con un'attenzione, in anticipo sui tempi, a complessi fenomeni socioculturali di interazione, ibridazione e resilienza ${ }^{1}$. Campo privilegiato per la decodificazione di tali rapporti è stato lo studio dei contesti, sia funerari (le necropoli

${ }^{1}$ GRASSI 1991a; GrASSI 1991b; GRASSI 1995; GRASSI 2000. 
di area insubre), che di abitato (Angera e Calvatone), con un approfondimento decisivo nei confronti della ceramica, sia da mensa (la vernice nera su tutte) $)^{2}$, che da cucina.

In questo contributo, che vuole essere un omaggio alle sue appassionate intuizioni e ai suoi rigorosi studi, intendiamo riprendere il tema delle ceramiche d'uso domestico diffuse in area padana tra la seconda età del Ferro e la prima età imperiale (Fig. 1) caratterizzate da quella varietà nel repertorio esornativo evocata nella citazione in apertura, quell'«interesse vivissimo per la decorazione delle superfici, sulle quali la luce viene fatta vibrare portando al dissolvimento di ogni consistenza tettonica

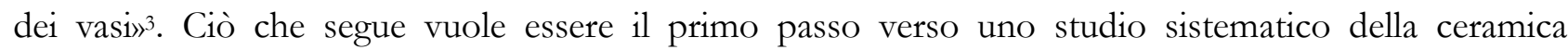
domestica di area padana della seconda metà del I millennio a.C. Un'esigenza legata alla constatazione che in questo ambito cronologico, territoriale e culturale «la recherche sur la vaisselle céramique est restée épisodique, plutôt marginale et aux contours encore mal définis» ${ }^{4}$, con studi perlopiù di taglio locale e sul breve periodo. In questa sede ci limiteremo a tratteggiare lo stato dell'arte, esplicitando i principali problemi di definizione terminologica, di ricostruzione dei processi tecnologici e di inquadramento cronologico, per accennare, infine, a possibili implicazioni storiche e culturali e a prospettive di ricerca.

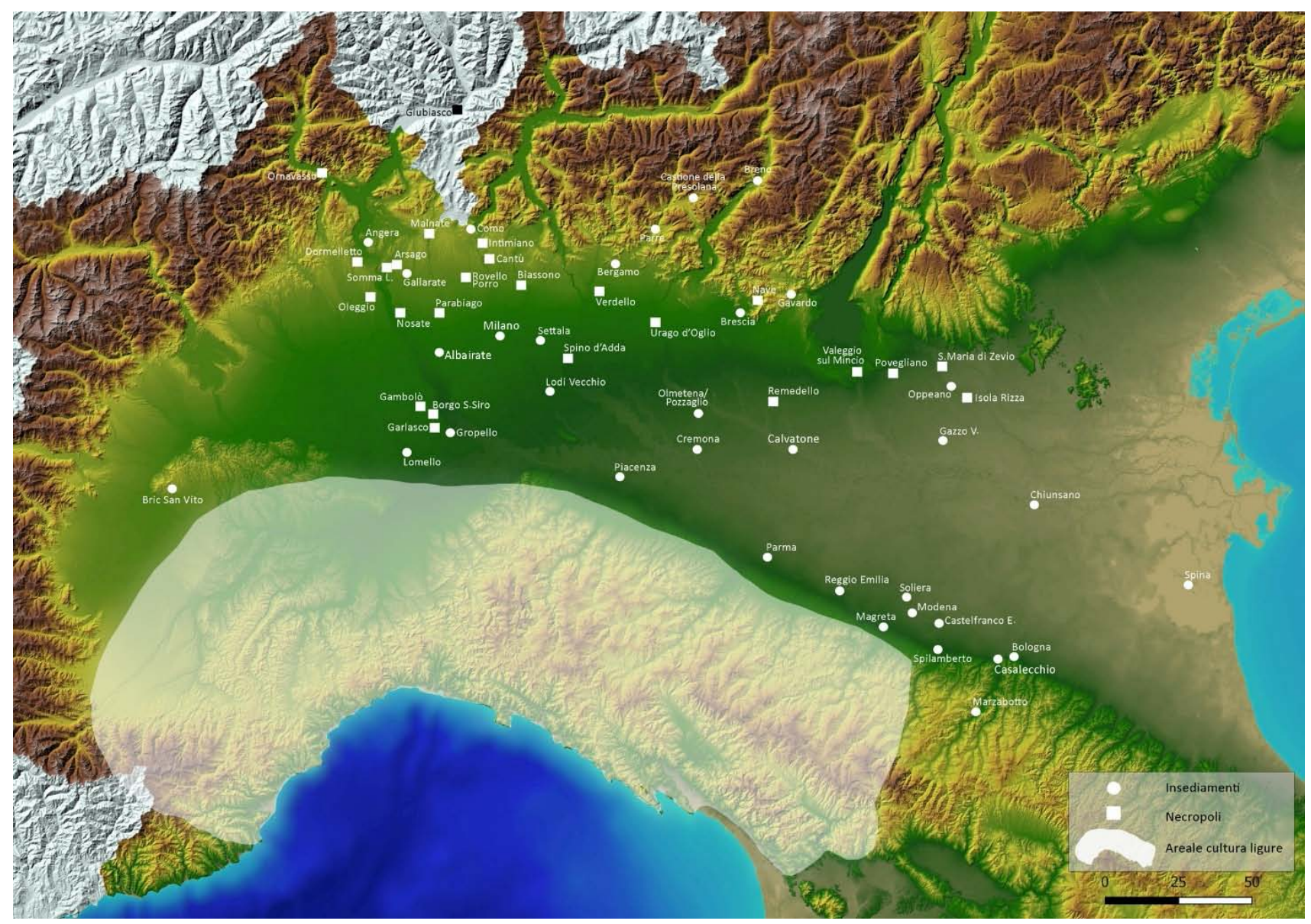

Fig. 1. L'areale cisalpino centrale (Piemonte orientale, Lombardia, Veneto occidentale, Emilia) interessato dalla presenza di ceramica d'impasto decorata tra la fine del V sec. a.C. e la romanizzazione, con le principali attestazioni in contesti d'abitato e funerari (elaborazione Autore).

\footnotetext{
${ }^{2}$ Grassi 2008.

3 ARSLAN 1971, p. 70; cfr. Grassi 2000, p. 19, nota 3.

4 Vitali 2014a, p. 296.
} 


\section{Il riconoscimento come classe e il problema del nome}

\subsection{Storia degli studi}

Il ritrovamento, casuale o frutto di episodici interventi di scavo, di ceramiche domestiche con decorazioni che esulavano dal repertorio formale romano attirò sporadicamente l'attenzione dei pionieri dell'archeologia cisalpina. Antonio Giussani, ad esempio, negli anni '30 annotava l'«ornamentazione semplice, ma elegante» di due «vasi decorati a pigna» in corredi da Caversaccio nel Comasco $^{5}$. Nel dopoguerra Guido Sutermeister si soffermava su un'olletta decorata proveniente da una delle necropoli di Parabiago da lui esplorate, facendone analizzare l'impasto e speculando sulle possibili funzioni: «un crogiolo» oppure una «pentola da cucina» ${ }^{6}$ (Fig. 2).

Per una svolta decisiva si devono attendere gli anni Settanta: da un lato le edizioni sistematiche delle necropoli del Canton Ticino fornivano associazioni di contesto e inquadramenti crono-tipologici in cui entravano a pieno diritto le ceramiche ${ }^{7}$. Più a sud, lungo l'asse del Ticino, le pubblicazioni dei complessi funerari e produttivi della Lomellina (Garlasco, Gropello Cairoli) ${ }^{8}$ davano al contempo un contributo decisivo per l'avvio degli studi sul cosiddetto "celtismo cisalpino"”.

口

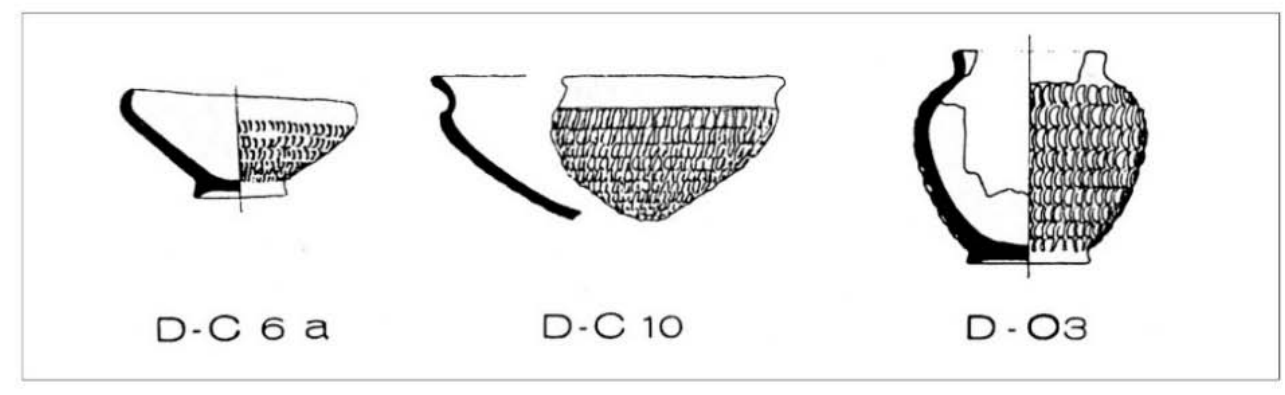

1
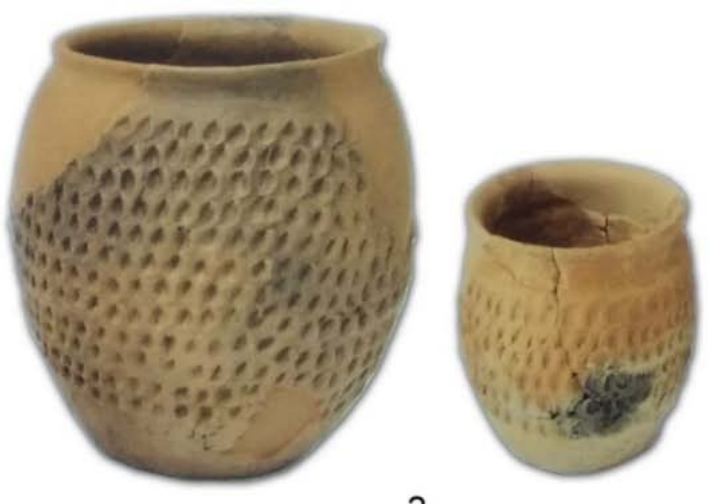

2

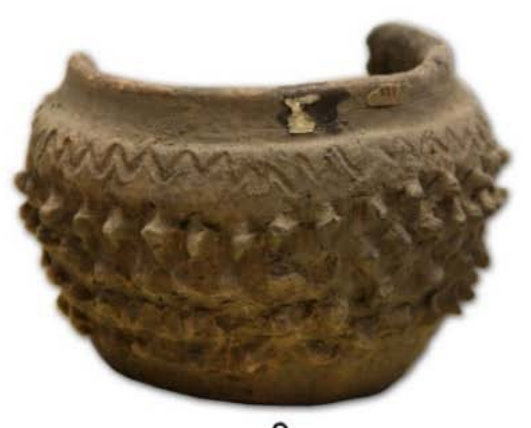

3

Fig. 2. 1 ceramiche da Garlasco (da ARSLAN 1977); 2 ollette da San Giorgio su Legnano (CMIC, Museo Civico Guido Sutermeister); 3 "olletta celtica", Museo Archeologico di Milano (CWikiCommons). Non in scala.

\footnotetext{
5 Cfr. Grassi 2000, p. 21, nota 20, con riferimento.

${ }^{6}$ SUTERMEISTER 1960; cfr. VOLONTÈ 1996, p. 35, nota 77, tav. 15.2.

7 Piana AgostinetTi 1972; Graue 1974; StÖCKLi 1975.

8 Arslan 1970-73; ArsLan 1971; ArSLAN 1972; Fortunati ZuCCÀla 1979; TrucCo 1979. Allo stesso tempo Gloria Vannacci Lunazzi pubblicava le tombe di Remedello Sotto e di Ca' di Marco (BS) (VANNACCi LunAZZI 1977). Una revisione dei dati funerari della Lomellina è ora in SCHEFFLER 2018.

9 ArSLan 1971-74; ArSLAN 1977.
} 
Riguardo le ceramiche, Ermanno Arslan metteva per la prima volta in evidenza non solo le produzioni fini (vernice nera locale, vasi a trottola, ceramica grigia), ma anche quella che definiva "ceramica a bugnette" (Fig. 2): «Di produzione quasi sempre domestica sono i tipi cd. "a bugnette". Si tratta di vasi, di forma variabile (solitamente però ollette e ciotole profonde) e di dimensioni variabili, in impasto mal depurato e spesso malcotto, plasmati quasi sempre a mano, coperti da una decorazione a tappeto di tacche e creste, qualche volta con una organizzazione ad alveare abbastanza regolare, altre volte con disposizione irregolare di linee parallele. Una fascia sotto il labbro e sul piede è solitamente risparmiata dalla decorazione. La diffusione del tipo copre l'intera area celtica e celtizzata» ${ }^{10}$. Egli fonda su queste ceramiche anche alcune considerazioni circa il tenore sociale delle popolazioni locali e il riconoscimento di facies locali che, nel corso della romanizzazione, si sarebbero via via uniformate (aspetti sui quali torneremo più avanti).

Di lì a poco, durante gli anni Ottanta, si assiste all'edizione di scavi e di cataloghi di materiali lombardi del periodo tardo La Tène ${ }^{11}$ e, grazie soprattutto a Raffaele de Marinis, alla sistemazione pressoché definitiva delle crono-tipologie e della periodizzazione della seconda età del Ferro in Italia settentrionale ${ }^{12}$.

È però con gli anni Novanta, anche a seguito dell'esponenziale aumento di scavi urbani, d'emergenza e di ricerca avviati nel decennio precedente ${ }^{13}$, che la ceramica domestica diventa protagonista di approfondimenti e proposte classificatorie: al 1991 risale l'edizione degli scavi della linea metropolitana 3 di Milano, con l'esaustiva pubblicazione della ceramica comune ${ }^{14}$, cui seguono importanti contributi sull'abitato di Angera ${ }^{15}$ e sul vicus di Calvatone/Bedriacum ${ }^{16}$. I materiali editi fino ad allora confluiscono quindi nel lavoro di sistemazione tipologica della ceramica lombarda coordinato nel 1998 da Gloria Olcese ${ }^{17}$.

In quegli stessi anni si devono a Maria Teresa Grassi, come ricordato all'inizio, lavori di sintesi sul celtismo cispadano come fenomeno storico $^{18}$, e sulla romanizzazione degli Insubri in particolare ${ }^{19}$, nei quali la ceramica decorata assume particolare valore in termini culturali (infra).

Un fondamentale incremento nella documentazione disponibile viene inoltre dall'edizione di alcune necropoli tra Lombardia e Piemonte orientale (Arsago Seprio, Parabiago, Oleggio, Dormelletto $^{20}$ e in provincia di Verona ${ }^{21}$.

Con gli anni Duemila la conoscenza sulle ceramiche domestiche della seconda età del Ferro si amplia ai territori a sud del Po. Sostanzialmente ignorata fino ad allora (e persino confusa con materiali

\footnotetext{
10 ARSLAN 1977, p. 474. Ceramica domestica con "intaccature digitali" veniva segnalata anche da Mario Degani a Reggio Emilia (cfr. BIONDANI 2014, p. 234, nota 18).

11 In particolare, i lavori di Marco Tizzoni (Tizzoni 1981; Tizzoni 1982; Tizzoni 1984; Tizzoni 1985). Da ricordare inoltre gli scavi a Como-Pianvalle (NEgroni CATACCHIO 1982), nella necropoli di Somma Lombardo (SimONE 1986), e a Milano-S. Maria alla Porta (CERESA MORI - TiZZONi 1986).

12 DE MARINIS 1977 («la première synthèse approfondie consacrée à la Cisalpine gauloise», nelle parole di VITALI $2014 \mathrm{a}$, p. 296); DE MARINIS 1986. Periodizzazione che accantona il precedente tentativo in NEGRONI CATACCHIO 1975.

13 Ad esempio Brogiolo 1984.

14 GUGLIELMETTI et alii 1991.

15 Domanico 1995.

16 Della Porta - SFredda 1996.

17 Della PORTA et alii 1998.

18 GRASSI 1991a.

${ }^{19}$ GRASSI 1991b; GRASSI 1995.

20 Arsago 1990; Antichi silenzi 1996; Spagnolo Garzoli 1999; SPAGNOLO GarZOLi 2009.

21 SALZANi 1995; SALZANi 1996; SALZANi 1998.
} 
dell'età del Bronzo ${ }^{22}$, con l'eccezione di Piacenza ${ }^{23}$, questa classe è emersa con decisione grazie alla pubblicazione degli scavi dell'insediamento di Casalecchio di Reno ${ }^{24}$. Altri frammenti sono stati in seguito riconosciuti al Forte Urbano di Castelfranco Emilia e in vari siti mappati per gli atlanti del territorio modenese ${ }^{25}$, oltre che a Bologna ${ }^{26}$, Spina ${ }^{27}$, Reggio Emilia ${ }^{28}$ e Marzabotto ${ }^{29}$. A Daniele Vitali dobbiamo un importante inquadramento del problema nell'areale "boico"30.

Rilevanti contributi hanno riguardato anche l'areale ligure ${ }^{31}$ che, come vedremo, si configura come uno snodo cruciale per la classificazione e la diffusione delle ceramiche domestiche decorate.

A nord del Po, in anni recenti, sono stati infine editi i materiali di Lodi Vecchio ${ }^{32}$, del Comasco ${ }^{33}$, del territorio di Varese $^{34}$, la necropoli di Giubiasco ${ }^{35}$, delle valli bergamasche e della Val Camonica ${ }^{36}$, i contesti di scavo di Calvatone ${ }^{37}$, di Brescia - santuario tardo-repubblicano e del suo territorio ${ }^{38}$, di Milano città e suburbio ${ }^{39}$, di Cremona - Piazza Marconi e degli insediamenti rustici del Cremonese ${ }^{40}$, mentre la pianura veronese è stata messa a sistema da Federico Biondani ${ }^{41}$.

\section{2 «Well, What Are We Going to Call It Now?» ${ }^{42}$}

$\mathrm{Al}$ di là della storia delle scoperte e delle edizioni, un primo nodo da sciogliere è quello del nome. Da una sommaria ricognizione della terminologia impiegata in letteratura (Tab. 1), si evince come, a fronte di un sostanziale accordo sull'individuazione di una classe con caratteristiche ben riconoscibili, le denominazioni siano alquanto eterogenee: partendo da un iniziale riferimento alla tecnica impiegata ("ceramica a bugnette", "bugnette ad alveare"), si sono in seguito adottati termini connessi all'ambito cronologico, per quanto connotato ("periodo tardo La Tène", "dell'età gallica"), fino al prevalere negli ultimi due decenni di più espliciti rimandi all'ambito culturale - e inevitabilmente etnico ("olle celtiche", "ceramiche celtiche", "ceramica tardoceltica"). Un crescente uso del concetto di 'tradizione'

\footnotetext{
22 Come avvenuto a Marzabotto con i materiali dal cd. Santuario Fontile (BuOITE - ZAMBONi 2008, p. 89; MORPURGO 2016 , p. 152, nota 122). Fraintendimenti di questo tipo non sono rimasti isolati (ad esempio il confronto proposto in CATTANEO 2003, p. 190, nota 31).

23 Marini Calvani 1990.

${ }^{24}$ Ferrari - Mengoli 2005; inoltre Vitali 2014a, pp. 298-301. Vedi anche le osservazioni in Lejars 2006.

25 Buoite - ZAmboni 2008. Una sintesi in Buoite - ZAmBoni 2012 (la sezione poster del convegno "Celti d'Italia" del 2010, è rimasta inedita; l'articolo è disponibile online in versione draft). Vedi anche LOCATELLI 2018, p. 27. Un lotto di ceramica d'impasto "di tradizione celtica" è emerso da scavi nell'insediamento pluristratificato di Spilamberto-via Macchioni, ma risulta al momento inedito (PANCALDI 2010).

26 TASSINARI 2010.

27 BuOITE - ZAMBONi 2013.

28 BIONDANI 2014.

29 Morpurgo 2016.

30 Vitali 2014a.

31 Tra i più recenti Gambari - Venturino Gambari 2004; FERrero et alii 2004; Ferrero 2007 (area tortonese); MARCHIARO 2012; MORDEGLIA 2016 (con riferimenti precedenti, inclusi Genova e la Liguria costiera).

32 Cattaneo 2003.

${ }^{33}$ RAPI 2009a; BUTTI RONCHETTI 2009-10; GIORGI et alii 2009-10 (tutti con ulteriore bibliografia).

${ }^{34}$ RAPI 2009b; RAPI 2009c; RAPI 2009d; RAPI 2009e.

35 TORI et alii 2010.

36 Poggiani Keller 2006; GuglielmetTi - Solano 2010, pp. 246-248.

37 GRASSI 2013.

38 RAgaZZI - SOlAno 2014. Per Urago d’Oglio: Rossi 2002; Grassi - MANGANi 2012.

${ }^{39}$ CASINI - TIZZONI 2015; CONSONNI et alii 2021. SimONe Zopfi et alii 2009; SimONE ZOPFi 2008 (saggio 2).

40 CeCCHini - Airoldi 2018; Mete - Ridolfi 2014.

41 BiONDANI 2013; BIONDANI 2018.

42 Ferguson 1980, pp. 23-24.
} 
("tradizione locale", "tradizione indigena”, “tradizione preromana”, "tradizione La Tène”, "tradizione ligure e lateniana francese") sembra implicitamente voler sfumare la coloritura etnica, per insistere piuttosto sul gusto e sulla trasmissione di stili e tecniche decorative.

Se i termini "tradizione locale" o "indigena", impiegati soprattutto per le fasi di incipiente o avviata romanizzazione, rimandano alla continuità con la dimensione alloctona, l'uso in alcuni siti emiliani di "tradizione non locale" pone al contrario l'accento su manifestazioni allogene rispetto alla fase precedente, etrusco-padana ${ }^{43}$.

\begin{tabular}{|c|c|c|c|c|}
\hline \multirow{2}{*}{ Riferimento } & \multirow{2}{*}{ Sito, territorio } & \multicolumn{3}{|c|}{ Definizione } \\
\hline & & 1. Tecnica & $\begin{array}{l}\text { 2. Ambito cronologico, } \\
\text { periodo }\end{array}$ & $\begin{array}{l}\text { 3. Ambito culturale e/o } \\
\text { stilistico ("tradizione") }\end{array}$ \\
\hline ARSLAN $1971 ; 1970-73$ & Lomellina (PV) & $\begin{array}{l}\text { "ceramica a } \\
\text { bugnette" }\end{array}$ & & \\
\hline VANNACCI LUNAZZI 1977 & $\begin{array}{l}\text { Remedello, } \\
\text { Fiesse (BS) }\end{array}$ & $\begin{array}{l}\text { "impressioni a } \\
\text { bugnette" }\end{array}$ & & \\
\hline MARINI CALVANI 1990 & Piacenza & & $\begin{array}{l}\text { "ceramica d'impasto } \\
\text { tardolateniana" }\end{array}$ & \\
\hline GUGLIELMETTI et alii 1991 & Milano & & & $\begin{array}{l}\text { "olle decorate di tradizione } \\
\text { celtica" }\end{array}$ \\
\hline DOMANICO 1995 & Angera (VA) & & $\begin{array}{l}\text { "ceramica decorata del } \\
\text { periodo tardo La Tène" }\end{array}$ & \\
\hline $\begin{array}{l}\text { VOLONTÉ 1996; CROCI } \\
1996\end{array}$ & Parabiago (MI) & & & $\begin{array}{l}\text { "ollette di tradizione celtica"; } \\
\text { "ceramica d'uso domestico" "con } \\
\text { decorazioni tardo La Tène" }\end{array}$ \\
\hline $\begin{array}{l}\text { DELLA PORTA - SFREDDA } \\
1996\end{array}$ & Calvatone (CR) & & $\begin{array}{l}\text { "ceramica comune" } \\
\text { "dell'epoca tardoceltica" }\end{array}$ & \\
\hline DELLA PORTA et alii 1998 & Lombardia & & & ceramica "tardo-celtica" \\
\hline ONGARO 1999 & Brescia & & "ceramica dell'età gallica" & \\
\hline POLETTI ECCLESIA 1999 & Oleggio (NO) & & & $\begin{array}{l}\text { "ceramica grossolana di } \\
\text { tradizione locale" }\end{array}$ \\
\hline GRASSI 2000 & Lombardia & & & $\begin{array}{l}\text { "produzione fittile di tradizione } \\
\text { celtica" }\end{array}$ \\
\hline GROSSETTI 2002 & $\begin{array}{l}\text { Pianello Val } \\
\text { Tidone (PC) }\end{array}$ & & & "ceramica di tradizione lateniana" \\
\hline CATTANEO 2003 & Lodi Vecchio & & $\begin{array}{l}\text { "ceramica comune tardo- } \\
\text { celtica" }\end{array}$ & \\
\hline FERRARI - MENGOLI 2005 & $\begin{array}{l}\text { Casalecchio di } \\
\text { Reno (BO) }\end{array}$ & & & $\begin{array}{l}\text { "ceramica d'impasto" "di } \\
\text { produzione locale" "ascrivibile } \\
\text { all'ambito culturale celtico" }\end{array}$ \\
\hline BUOITE - ZAMBONI 2008 & $\begin{array}{l}\text { Castelfranco } \\
\text { Emilia (MO) }\end{array}$ & & & $\begin{array}{l}\text { "ceramica d'impasto di tradizione } \\
\text { non locale" }\end{array}$ \\
\hline RATTO 2009 & Dormelletto (NO) & $\begin{array}{l}\text { "impasto } \\
\text { grossolano" }\end{array}$ & & \\
\hline BUOITE - ZAMBONI 2013 & Spina (FE) & & & $\begin{array}{l}\text { "ceramica d'impasto di tradizione } \\
\text { La Tène" }\end{array}$ \\
\hline GRASSI 2013 & Calvatone (CR) & & & $\begin{array}{l}\text { "ceramica decorata di tradizione } \\
\text { celtica" }\end{array}$ \\
\hline RAGAZZI - SOLANO 2014 & Brescia & & & $\begin{array}{l}\text { "ceramica comune grezza" "olle } \\
\text { celtiche" }\end{array}$ \\
\hline CASINI - TIZZONI 2015 & Milano & & & $\begin{array}{l}\text { "Recipienti di tradizione ligure e } \\
\text { latèniana francese" }\end{array}$ \\
\hline MORPURGO 2016 & Marzabotto & & & $\begin{array}{l}\text { "ceramica d'impasto di tradizione } \\
\text { non locale" }\end{array}$ \\
\hline CECCHINI - AIROLDI 2018 & Cremona & & & "ceramica di tradizione celtica" \\
\hline CROTTI 2019 & $\begin{array}{l}\text { Castelfranco } \\
\text { Emilia (MO) }\end{array}$ & & & $\begin{array}{l}\text { "ceramica di tradizione } \\
\text { preromana" }\end{array}$ \\
\hline
\end{tabular}

Tab. 1. Principali definizioni adottate in letteratura (Lombardia ed Emilia-Romagna), in ordine cronologico. I nomi sono distinti in base al loro riferirsi a: 1. tecnica; 2. cronologia; 3. cultura o stile.

43 Per i riferimenti bibliografici si veda Tab. 1, prima colonna. 
Altre definizioni recenti (non riportate in tabella) sembrano indirizzare verso una sintesi tra i due sistemi: "ceramica comune grezza della tarda età del ferro dell'area celtizzata"44, oppure "ceramica indigena decorata della romanizzazione" ${ }^{45}$.

La scelta di un nome non è mai un'operazione neutra. Nel caso di questa classe la difficoltà è data dal sovrapporsi di aspetti di natura sia cronologica che etnico-culturale. Conviene allora procedere per esclusione: dando per scontato che un mero riferimento alle decorazioni non sia sufficiente, $\mathrm{i}$ termini "grezza" e "comune" sono il primo impreciso ${ }^{46}$, il secondo riduttivo perché impiegato soprattutto nella tradizione di studi sulla ceramica romana. Quindi la categoria “impasto", per quanto generica, sembra la formulazione da preferire.

Più importante è riflettere sul problema dell'uso di termini come "lateniana", "gallica" o "celtica". Nessuno di questi appare adeguato, per ragioni diverse: da un punto di vista cronologico, infatti, l'uso del termine "La Tène" non coprirebbe tanto le fasi più antiche in cui queste ceramiche decorate compaiono (tardo Hallstatt in Europa centrale, periodo Ligure II, infra), tanto quelle più recenti di epoca romana, con attestazioni fino al periodo giulio-claudio.

Inoltre, se si passa alle implicazioni culturali, le etichette "celtico" o "gallico" sono da respingere a causa delle loro intrinseche implicazioni storico-culturali ed etniciste: come ha dimostrato John Collis ${ }^{47}$, quello celtico è un mito romantico costruito a posteriori, un revival dalle coloriture politiche fin troppo evidenti. Ad esso non corrisponde alcuna realtà archeologica, tantomeno un blocco monolitico di attributi, né fisici, né linguistici, né culturali. Allo stesso modo il termine "La Tène" «is not intended to be (as is sometimes the case) a politically correct whitewash for the concept of 'Celts' [...]. No one was ever born a Latènian.... ${ }^{48}$. Anche il riferimento a un vago mondo "indigeno" è improprio, in quanto limitato da una prospettiva colonialista, in questo caso di matrice romana.

È allora preferibile adottare una definizione generica, e del tutto convenzionale, come "ceramica d'impasto decorata" diffusa in Cisalpina tra la seconda età del Ferro e la romanizzazione. Non imporre un'etichetta troppo specifica, del resto, ci permette di evitare una falsa impressione di uniformità (temporale e geografica). Sarà di volta in volta necessario evidenziare specificità, somiglianze e differenze ${ }^{49}$, rispetto a un patrimonio di tecniche, gusti e tratti condivisi che appare dinamico e permeabile.

\section{Aspetti tecnologici e produttivi}

Dietro al nome che abbiamo proposto, inevitabilmente generico, si cela un ampio spettro di produzioni locali e regionali, distribuite su un lungo arco temporale, almeno dal V secolo a.C. fino alla prima età imperiale. A fronte di una scarsità di analisi mineralogiche e archeometriche disponibili ${ }^{50}$, si

\footnotetext{
44 BIONDANi 2013, p. 42.

45 SENA CHIESA 2014, p. 40, nota 24.

${ }^{46}$ Come già evidenziato in ZAMBONI 2016, p. 153, nota 607.

47 COLLis 2003.

48 KYSELA 2020, p. 19.

49 REMOTTI 2019.

50 ANGANUZZI et alii 1986; PREDIERI - SFRECOLA 1996; CALANDRA et alii 1998.
} 
presuppone comunemente un ricorrere ai bacini di approvvigionamento locale per argille, frazione minerale e smagranti ${ }^{51}$, senza tuttavia escludere a priori una mobilità di artigiani e prodotti ${ }^{52}$.

Sulla base della letteratura e dell'osservazione diretta di alcuni campioni, si può affermare che la tecnologia tenda a non impiegare il tornio, con un eventuale ricorso alla tournette ${ }^{53}$. Le superfici possono essere più o meno rifinite, a mano o a strumento, tramite lisciatura sommaria o lucidatura. La cottura avviene perlopiù a basse temperature, in strutture pirotecnologiche di non elevata complessità, quali fornaci a catasta o forni a camera singola.

L'esito formale è quantomai vario, con corpi ceramici a diverso grado di depurazione e un'ampia gamma di esiti cromatici (dal nero o grigio scuro, al bruno, al rosso-arancio), a volte con il "cuore nero" 54 (indice di una non accurata conduzione della cottura). L'impressione, non suffragata al momento da analisi tecnologiche o dati statistici, è che nelle prime fasi $(\mathrm{LT} B / \mathrm{C})$ la produzione sia più corsiva (manifattura a mano, superfici scarsamente rifinite, cotture poco controllate), per assumere caratteri di maggiore standardizzazione tra la fine dell'età del Ferro e la romanizzazione (impiego del tornio, superfici lucidate, cotture in impianti pirotecnologici strutturati).

Il panorama delle forme vascolari è ridotto e sostanzialmente ripetitivo, comprendendo, tra quelle chiuse, olle, ollette, bicchieri e boccali ansati. I corpi sono essenzialmente situliformi, ovoidi o globulari, con orli arrotondati o assottigliati che possono essere estroflessi, diritti, o rientranti. Una caratteristica distintiva di alcuni tipi di olle è la spalla distinta o accentuata da un gradino (Fig. 4, fasi LT C e D). Le forme aperte decorate, diffuse sul territorio ma statisticamente minoritarie, annoverano ciotole, o ciotole-coperchio, a vasca troncoconica con orli indistinti.

\subsection{Le sintassi decorative}

L'aspetto più evidente e caratteristico di questa classe è il ricco e variegato repertorio decorativo, che interessa soprattutto la spalla o gran parte della superficie esterna del vaso. A fronte di diverse proposte di ordinamento e classificazione delle tecniche decorative, perlopiù a livello locale, intra-sito ${ }^{55}$, è evidente come manchi una condivisione, anche di massima, su vocabolari e categorie di tecniche e sintassi ${ }^{56}$.

L'operazione non è banale, sia per la natura eterogenea, non standardizzata, della classe, ma anche per lo stato della documentazione disponibile ${ }^{57}$ : «Spesso viene fatta confusione tra le varie tipologie decorative e frequentemente la non elevata qualità dei disegni pubblicati non consente di distinguere tra impressioni di polpastrello e impressioni a unghiate o a bugnette» ${ }^{58}$.

Ciò che segue è perciò un tentativo preliminare e provvisorio di ordinamento su base sovraregionale delle principali tecniche e sintassi decorative adottate nelle produzioni in ceramica

\footnotetext{
51 Ad esempio Della Porta - SFredda 1996, p. 155, e RagazZI - Solano 2014, p. 68, per l'impiego in alcuni distretti territoriali di conchiglie fossili triturate.

52 GugLIELMETTI et alii 1991, p. 170.

${ }^{53}$ Guglielmetti et alii 1991, p. 169; Della Porta - SFredda 1996, p. 152; Ratto 2009, p. 179; Grassi 2000 , p. 19 (rifinitura di alcune parti del vaso, tra cui l'orlo, al tornio lento); CATTANEO 2003, p. 187; BIONDANi 2014, p. 236.

${ }_{54}$ Per la terminologia tecnica si faccia riferimento a LEVI 2010.

55 GuglielmetTi et alii 1991, p. 171; Domanico 1995, pp. 285-286; PoletTi Ecclesia 1999, pp. 303-304, fig. 340; CATtAneo 2003; RApi 2009a, p. 91; MARChiaro 2012, pp. 46-48, figg. 5-6. Con l'eccezione del lavoro di Lucia Mordeglia sulla ceramica ligure (MORDEGLIA 2016, pp. 254-255).

${ }^{56}$ Cfr. ONGARO 1999, pp. 43-44.

${ }^{57}$ GRASSI 2000, p. 20.

${ }^{58}$ DE MARINIS 2019, p. 47. Anche SCHINDLER-KAUDELKA - BIONDANi 2018, nota 21.
} 
d'impasto tra seconda età del Ferro e primo periodo imperiale in Cisalpina. Non viene preso direttamente in considerazione l'ambito ligure (Liguria, Piemonte meridionale, Appennino toscoemiliano, Fig. 1) che, come detto, è stato recentemente oggetto di un'esaustiva sistematizzazione ${ }^{59}$.

\begin{tabular}{|c|c|c|c|c|}
\hline Tecnica & $\begin{array}{c}\text { Stato di } \\
\text { essiccazione }\end{array}$ & Strumento & Movimento & $\begin{array}{l}\text { Definizioni } \\
\text { precedenti }\end{array}$ \\
\hline I. Incisione & Cuoio o verde & $\begin{array}{l}\text { Punta; } \\
\text { pettine; }\end{array}$ & $\begin{array}{l}\text { Ripetuto, } \\
\text { continuo }\end{array}$ & $\begin{array}{l}\text { Solcatura; } \\
\text { taglio; } \\
\text { linee incise; } \\
\text { fasce di impressioni }\end{array}$ \\
\hline \multirow{2}{*}{ II. Impressione } & Verde o semi-cuoio & $\begin{array}{l}\text { Punta, } \\
\text { pettine, } \\
\text { spatola, } \\
\text { stampo }\end{array}$ & $\begin{array}{l}\text { Singolo ripetuto; } \\
\text { continuo interrotto }\end{array}$ & $\begin{array}{l}\text { Tacche; } \\
\text { scanalature; } \\
\text { registri }\end{array}$ \\
\hline & Verde & $\begin{array}{l}\text { Polpastrello e unghia; } \\
\text { polpastrello }\end{array}$ & $\begin{array}{l}\text { Singolo, } \\
\text { singolo ripetuto; } \\
\text { interrotto }\end{array}$ & $\begin{array}{l}\text { Alveare; } \\
\text { tipo Rossiglione }\end{array}$ \\
\hline III. Plastica & Verde & Mano & $\begin{array}{l}\text { Singolo ripetuto: } \\
\text { applicazione o } \\
\text { pizzicatura }\end{array}$ & $\begin{array}{l}\text { Bugnette, bugne; } \\
\text { tubercoli, protuberanze }\end{array}$ \\
\hline
\end{tabular}

Tab. 2. Schema terminologico delle tecniche decorative presenti sulla ceramica d'impasto in Cisalpina: stato di essiccazione, strumenti, movimenti, e corrispondenze con definizione precedenti.

Una prima suddivisione riguarda le tecniche e i gesti impiegati per la realizzazione delle decorazioni, tra i quali distinguiamo tre principali categorie: incisioni, impressioni, e decorazioni plastiche (Tab. 2; Fig. 3).

Con "incisione" ${ }^{\prime 00}$ s'intende un'azione, tendenzialmente ripetuta, eseguita sulla superficie (il più delle volte allo stato cuoio $)^{61}$ tramite uno strumento a punta, con una pressione seguita dal trascinamento della punta stessa (movimento continuo). Lo strumento impiegato può essere una punta singola, con movimenti ripetuti a mano libera, oppure un pettine ${ }^{62}$. I motivi più frequenti sono lineari, ondulati, a zig-zag (continuo o spezzato), a spina di pesce, a reticolo, o a campiture rese a pettine ${ }^{63}$ (Fig. 3, I.1-6).

\footnotetext{
59 Mordeglia 2016.

${ }^{60}$ Motivi definiti altrove anche "solcature" (ad esempio DOMANICO 1995), o "tagli" (GUGLIELMETTI et alii 1991).

${ }^{61}$ Solo avendo a disposizione foto o disegni di alta qualità è possibile capire quali decorazioni vengano eseguite quando l'impasto è ancora fresco (stato verde), o piuttosto durante il processo di essiccazione (stato cuoio, o semi-cuoio). Ad esempio, grazie alle foto di dettaglio in RAGAZZI - SolANO 2014, tav. XL, si apprezza come le incisioni lineari del n. 3 siano eseguite all'inizio del processo di essiccazione, mentre quelle al n. 6 (ivi) sembrano piuttosto realizzate allo stato cuoio. Più in generale sarebbero necessarie prove di archeologia sperimentale per testare i diversi esiti e ricostruire dettagliatamente $i$ processi di manifattura.

${ }^{62}$ La distinzione tra linee tracciate singolarmente a mano libera e ripetute, e i fasci di linee resi con un pettine, richiede una documentazione grafica estremamente accurata (che evidenzi micro-differenze tra le singole linee o i fasci di linee), soprattutto quando la presa visione diretta del pezzo non è possibile. Lo stesso vale per alcune delle impressioni, soprattutto le teorie di punti.

L'esistenza di differenti tradizioni grafiche in uso tra scuole diverse (protostoria e archeologia classica) complica la situazione: in particolare va rilevato il fatto che chi si occupa di ceramica del periodo romano, rispetto agli specialisti di età del Ferro, tendenzialmente non usa il puntinato e le ombreggiature. Una lacuna che il più delle volte limita la descrizione e la comprensione dei dati (si veda, ad esempio, la resa non accurata del frammento bedriacense n. 223 in DELLA PORTA SFREDDA 1996, rispetto alla foto del medesimo, ivi., fig. 150).

${ }^{63}$ Il pettine trascinato (Fig. 3, I.6), peraltro non molto diffuso, è in realtà una via di mezzo tra un'incisione e un'impressione: lo strumento infatti incide la superficie (allo stato verde) e imprime un movimento continuo ripetuto, con un esito simile a quello della spatola nelle impressioni strumentali (Fig. 3, II.10).
} 


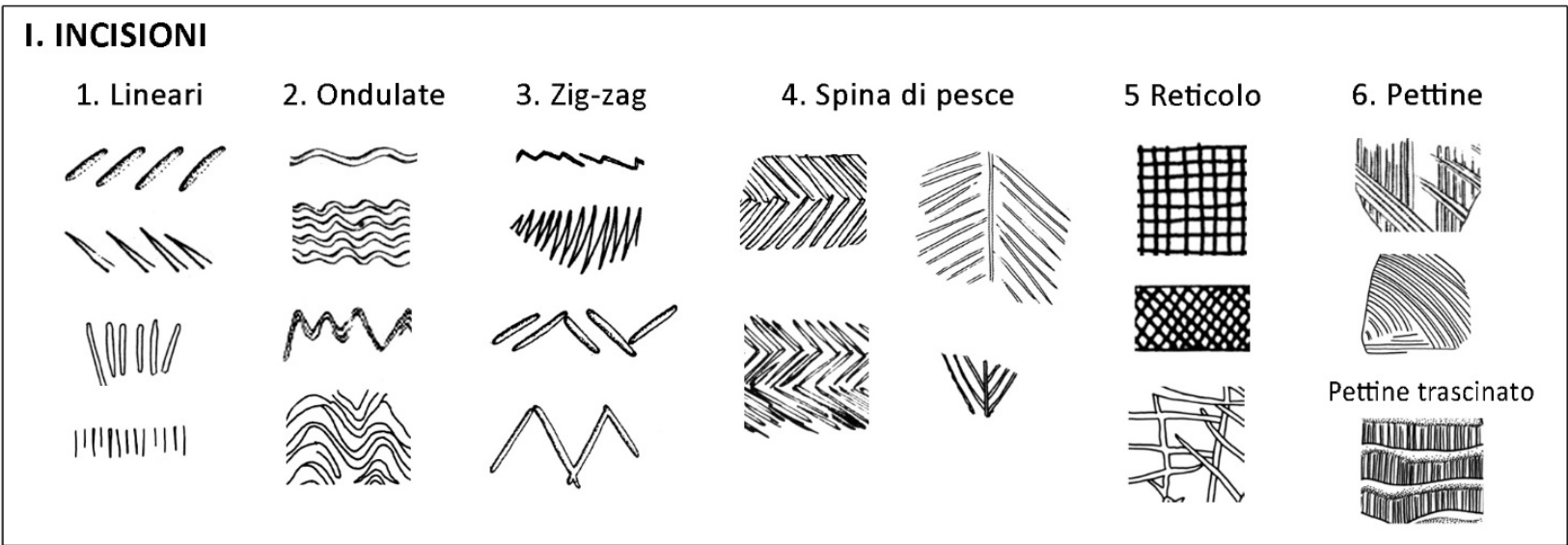

II. IMPRESSIONI STRUMENTALI

\begin{tabular}{|c|c|c|c|c|c|c|}
\hline 1. Punti & 2. Circolari & 3. Ovali & 4. Quadrangolari & 5. Rettangolari & 6. Triangolari & 7. Virgole \\
\hline$\therefore \circ$ & 0000 & 0000 & 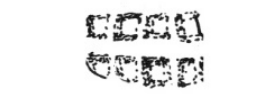 & $\begin{array}{l}0000 \\
0000\end{array}$ & $\therefore$ & $D D D 2$ \\
\hline !n!!! & $\begin{array}{l}000 \\
0000 \\
0000\end{array}$ & $\begin{array}{l}\text { exces } \\
\text { ecen }\end{array}$ & 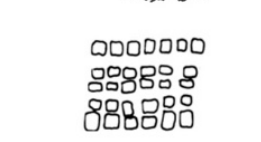 & $\begin{array}{l}00000000 \\
0000000 \\
0000000 \\
000000\end{array}$ & $\begin{array}{l}\square= \\
-\infty \\
0-5\end{array}$ & $\begin{array}{c}\triangle \Delta \triangle \Delta D \\
\text { 8. Semilune }\end{array}$ \\
\hline है। & 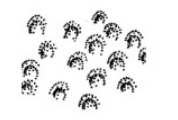 & 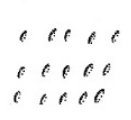 & $\theta: \theta 00$ & 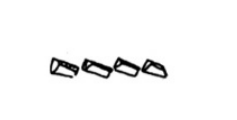 & 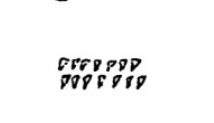 & 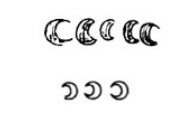 \\
\hline
\end{tabular}

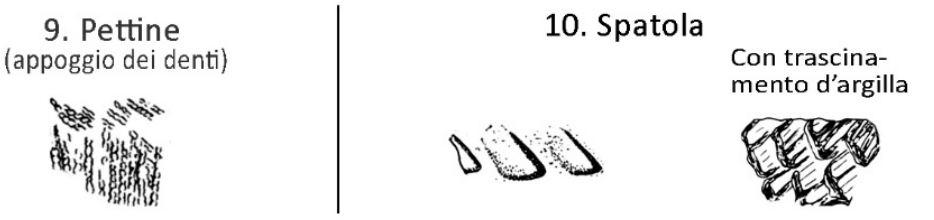

11. Falsa cordicella

$B A B B^{\circ}$

III. IMPRESSIONI DIGITALI

\begin{tabular}{|c|c|c|c|}
\hline & 1. Unghiate & $\begin{array}{c}\text { Con trascinamen } \\
\text { d'argilla }\end{array}$ & 2. Polpastrelli \\
\hline 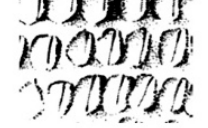 & $\leftrightarrow=$ & 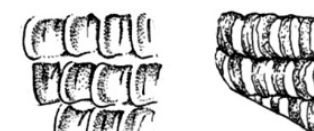 & Co \\
\hline 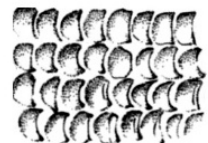 & 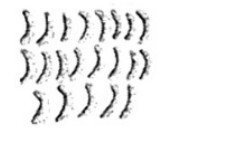 & 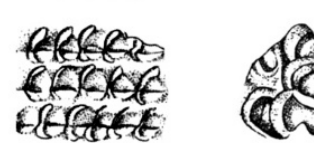 & 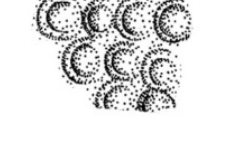 \\
\hline
\end{tabular}

IV. DECORAZIONI PLASTICHE

1. Bugne

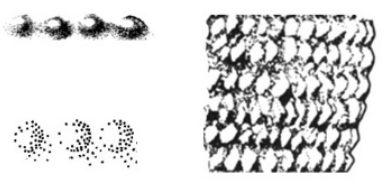

2. "Punta di diamante"

3. Quadrati, rombi

Fig. 3. Schema delle principali decorazioni attestate sulla ceramica d'impasto della seconda età del Ferro e della romanizzazione in Cisalpina (elaborazione Autore). 
L'"impressione" ${ }^{\prime \prime 4}$ invece avviene sulla superficie ceramica perlopiù allo stato verde, con uno strumento ("impressione strumentale", punta singola, pettine, spatola, stampo), o con un dito ("impressione digitale"). La prima prevede l'impiego di punte singole e punzoni con terminazioni di varia foggia: circolari, ovali quadrangolari, rettangoli, triangoli, virgole, semilune (Fig. 3, II.1-8). È probabile che gli strumenti fossero in materiali vari (cannucce, stecche, bastoncini in legno, in osso, eventualmente metallo). Il pettine in questo caso non viene trascinato ma appoggiato (Fig. 3, II.9). Compaiono anche strumenti a terminazione larga come spatole, anche trascinate (Fig. 3, II.10), e stampi (falsa cordicella).

L'“impressione digitale" viene ottenuta con unghiate ripetute, a volte con un breve trascinamento dell'argilla (movimento interrotto) (Fig. 3, III.1), oppure eseguendo una certa pressione del polpastrello su un unico punto (Fig. 3, III.2).

Le decorazioni "plastiche", infine, prevedono l'applicazione di elementi di argilla che sporgono dalla superficie quali bugne (a profilo regolare, arrotondato o appuntito, più o meno sviluppate), o tubercoli, "punte di diamante", e altri elementi di forma irregolare (Fig. 3, IV). È possibile che in alcuni casi l'effetto sia anche ottenuto tramite la "pizzicatura" (sollevamento e torsione dell'argilla tra pollice e indice) della superficie non ancora rifinita.

Tra le decorazioni plastiche rientrano naturalmente anche i cordoni applicati, che tuttavia in questa sede non vengono presi in considerazione dal momento che appaiono scarsamente attestati nelle regioni transappenniniche, mentre sono ampiamente diffusi in ambito ligure ${ }^{65}$. Anche un tipo particolare di decorazione a cordoncini con impressioni, definita convenzionalmente "stile di Kalenderberg" " è nota solo in siti afferenti alla sfera culturale ligure, e risulta assente altrove in Italia settentrionale.

Sempre in questa categoria di decorazioni applicate rientrerebbe la tecnica à sapin: un motivo plastico realizzato con file parallele di elementi triangolari che ricordano delle squame di pigna. Questa tecnica, tuttavia, non risulta al momento attestata a sud delle Alpi: «Alla luce di quanto finora pubblicato, ceramica con decorazione à sapin secondo la definizione di Mariën non è presente nella ceramica d'impasto ligure o della Transpadana e quindi sarebbe bene abbandonare questa denominazione per quanto riguarda l'Italia» ${ }^{67}$. Poco nota in pianura padana è infine la resa delle superfici scabra, o "rusticata", che in alcuni esemplari copre gran parte del vaso lasciando a risparmio l'orlo ${ }^{68}$.

Nonostante siano statisticamente preponderanti i vasi che, sulla spalla o su tutto il corpo, recano una sola teoria di decorazione, non mancano varie combinazioni e associazioni di motivi ornamentali. Quelle più frequenti vedono le incisioni, soprattutto lineari, a zig-zag, o a reticolo, assieme a impressioni strumentali (soprattutto ovali, quadrangolari, triangolari); oppure registri diversi di impressioni

\footnotetext{
${ }^{64}$ I medesimi esiti sono definiti altrove "tacche" (ad esempio GUGLIELMETTI et alii 1991; DOMANICO 1995; POLETTI ECCLESIA 1999). Se disposti in maniera da ricoprire gran parte del vaso sono chiamati anche "ad alveare" (supra, e SCHINDLER-KAUDELKA - BIONDANI 2018, p. 246).

${ }^{65}$ Mordeglia 2016, pp. 254-257.

66 Mordeglia 2016, pp. 254-255. Raffaele de Marinis ha però puntualizzato come «La comparsa di questa tecnica decorativa su amplissime aree geografiche e in epoche cronologicamente distanti, le toglie valore comparativo e la dizione “decorazione di stile Kalenderberg” deve essere considerata puramente convenzionale» (DE MARINIS 2019, p. 48).

${ }^{67}$ DE MARINIS 2019, p. 50.

68 Buoite - ZAMBOni 2012, fig. 7.
} 
strumentali; oppure ancora incisioni abbinate a impressioni digitali o a decorazioni plastiche (come, ad esempio, le incisioni ondulate e le punte di diamante in esemplari milanesi, Fig. 2.3, Fig. 4).

È probabile che specifiche combinazione di tecniche e motivi siano caratteristiche di certi periodi $^{69}$ e di alcune facies culturali: è già stato osservato, ad esempio, che l'abbinamento tra incisioni lineari e impressioni digitali a unghiate sia diffuso nella Lombardia orientale durante fasi più tarde, a partire dal I sec. a.C. ${ }^{70}$.

\section{Cronologia e distribuzione}

A questo punto è necessario chiedersi quando fanno la loro comparsa a nord degli Appennini i vasi e le sintassi decorative che abbiamo descritto. È possibile tratteggiare una mappa della loro distribuzione e seguire gli sviluppi della loro evoluzione formale? Va premesso che una definizione cronologica di dettaglio per queste ceramiche è tutt'altro che agevole, sia per la loro natura poco standardizzata, sia soprattutto per la scarsità di contesti chiusi e associazioni con altri materiali ben databili, sia d'abitato che di necropoli. Per quanto riguarda queste ultime, inoltre, è noto come durante la fase La Tène B (IV - metà III sec. a.C.) il rito funerario preveda la pressoché sistematica esclusione della ceramica dai corredi ${ }^{71}$.

\subsection{Dal Golasecca III A al La Tène C1}

Tra le poche sequenze insediative note, lo scavo di Milano - via Moneta ha restituito una notevole quantità e varietà di ceramica d'impasto decorata. Purtroppo si tratta in larga parte di contesti in giacitura secondaria, sconvolti dagli interventi urbanistici delle fasi storiche, per cui le considerazioni che seguono andranno verificate sulla base di altri contesti chiusi. Secondo la scansione proposta dagli editori (Fig. 4), in via Moneta i primi frammenti di olle in impasto decorate con incisioni lineari o a zigzag compaiono presto, in unità stratigrafiche datate tra la fine del V e i primi del IV sec. a.C. (fasi Golasecca III A2, III A3 $)^{72}$. Le attestazioni aumentano sensibilmente già con le successive fasi La Tène B1 e B2, quando accanto a olle ovoidi ancora legate alla tradizione precedente (orli a fascia e ingrossati) e decorate sulla spalla con file di incisioni lineari e a zig-zag, compaiono olle ovoidi e situliformi con impressioni circolari, ovali, fitte incisioni a pettine e, forse, impressioni digitali a unghiate ${ }^{73}$.

A Brescia, nell'area del futuro Capitolium, l'impasto decorato è documentato in contesti di scavo databili a partire dal III sec. a.C. ${ }^{74}$. In una prima fase (III-inizi II secolo) sono caratteristiche le olle ovoidi con orli estroflessi, arrotondati o ingrossati, decorate da incisioni lineari o più frequentemente, da file di impressioni strumentali: soprattutto quadrangolari, rettangolari, virgole, semilune ${ }^{75}$.

\footnotetext{
${ }^{69}$ In ambito ligure l'associazione tra impressioni digitali con trascinamento d'argilla ed elementi a zig-zag è «uno dei motivi decorativi caratterizzanti la ceramica indigena di IV secolo a.C. della Liguria interna e costiera» (MARCHIARO 2012, p. 47).

70 Della Porta - SFredda 1996 n. 222; RAgazZi - Solano 2014, tav. XXIX, n. 4.

71 Grassi 1991; 1995; Vitali 2014b. Nel presente lavoro, alla Fig. 4, la comparazione tra contesti di abitato (Milano, via Moneta) e di necropoli (Dormelletto) è intesa come una mera esemplificazione a fini di un inquadramento cronologico preliminare, e non implica parallelismi di altra natura tra due sfere culturalmente distanti.

${ }^{72}$ CASINI - Tizzoni 2015, p. 85, nota 66: la datazione si basa però sulle associazioni, mentre la giacitura è purtroppo secondaria.

73 CASINI - TiZZONI 2015, pp. 86-121, figg. 14-44.

74 RAGAZZI - SOLANO 2014, pp. 71-73.

75 RAGAZZI - SOLANO 2014, tavv. XXV-XXVIII.
} 
$\square$

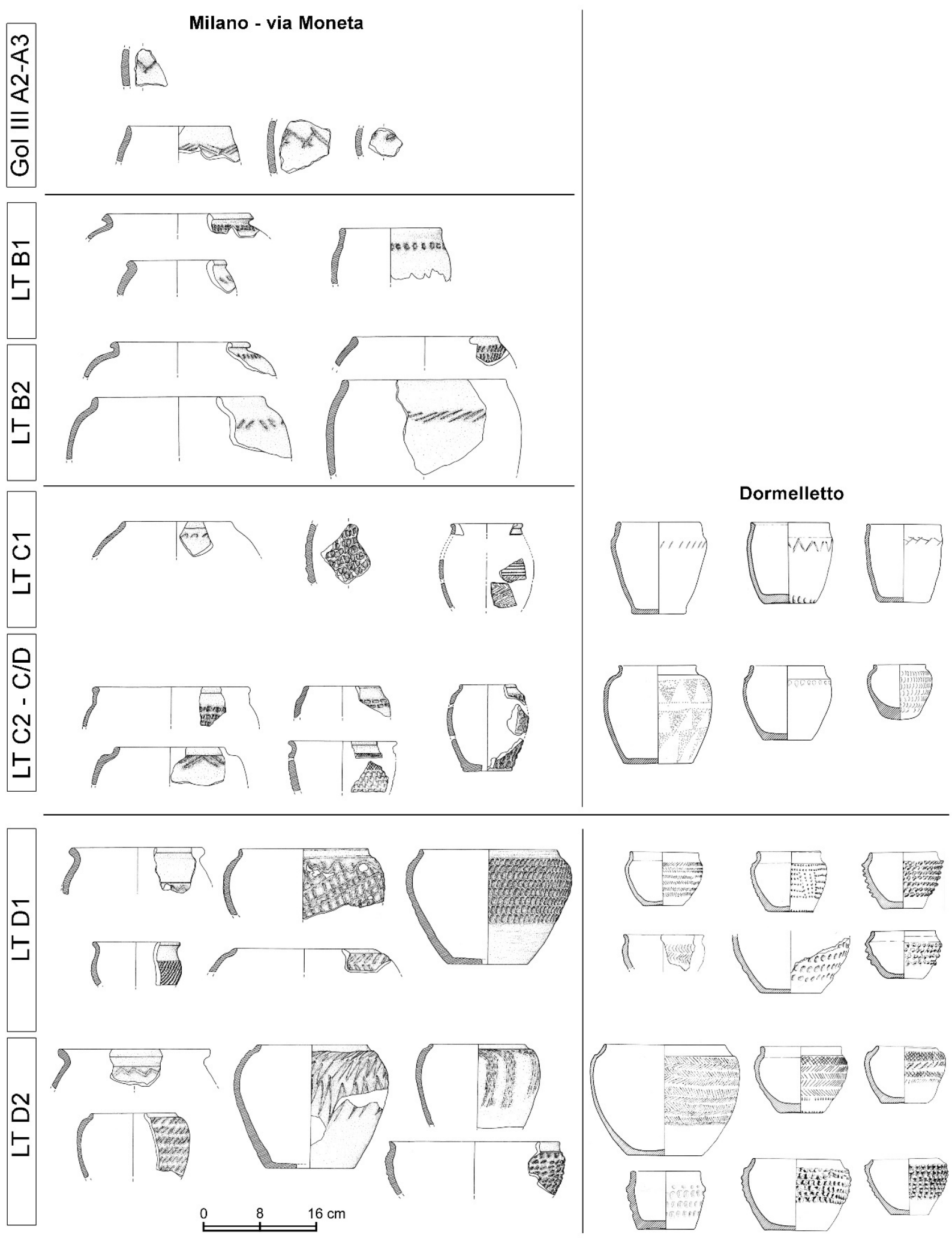

Fig. 4. Forme e decorazioni tra le fasi Golasecca IIIA2-IIIA3 e La Tène D2 in Lombardia, sulla base della sequenza di abitato di Milano - via Moneta, e dei corredi di Dormelletto (disegni da CASINI - TIZZONI 2015, e RATTO 2009; elaborazione Autore). 
Questi elementi (orli estroflessi e impressioni strumentali) proseguono con poche modifiche (un infittirsi delle campiture) fino all'età tardo-repubblicana ${ }^{76}$, andando a configurare un aspetto tipico della facies locale, "cenomane", di Brescia 77 .

Nella pianura emiliana la situazione è speculare, pur con alcune differenze. La ceramica d'impasto a mano decorata con teorie estranee al patrimonio etrusco-padano compare tra IV e prima metà del III sec. a.C., sia nelle fasi finali di insediamenti precedenti, come Spina, il Forte Urbano di Castelfranco Emilia, e Marzabotto ${ }^{78}$, o in siti riattivati dopo le invasioni storiche, quali Casalecchio di Reno o Bologna-via Andrea Costa ${ }^{79}$. In generale, in questa prima fase sono attestate incisioni lineari, a zig-zag e ondulate allineate sulla spalla delle olle, ma si nota, rispetto alla Lombardia, una precoce apparizione delle fitte impressioni digitali a unghiate e delle campiture a pettine, già a partire dal IV sec. a.C. ${ }^{80}$.

In Emilia il sito chiave per questa fase è certamente Casalecchio di Reno: in connessione con una necropoli che presenta materiali e rito inumatorio di evidente matrice lateniana ${ }^{81}$, l'area di insediamento ha restituito un'ingente quantità di olle decorate sulla spalla da incisioni lineari o a zig-zag, ma anche da impressioni a file di punti o ovali, e da fitte impressioni digitali a unghiate. La colmatura della fossa dalla quale provengono la maggior parte dei frammenti è datata al terzo quarto del III sec. a.C. (LT C1) ${ }^{82}$.

\subsection{Dal La Tène C2 alla prima età imperiale}

Come più volte sottolineato, il momento di fioritura di questo gusto decorativo è tuttavia da porre tra la fine dell'età del Ferro e la romanizzazione, tra la seconda metà del II e il I sec. a.C. ${ }^{83}$. Per questo periodo, in cui la ritualità funebre riammette la ceramica tra le offerte, le necropoli offrono associazioni di corredo datanti, mentre negli insediamenti, per quanto numerosi, rimangono rare le stratigrafie affidabili, e spesso la ceramica d'impasto decorata proviene da giaciture secondarie o da strati sconvolti.

A Milano - via Moneta dalla fase LT C/D aumentano le olle con breve orlo a colletto decorate con fitte campiture a impressioni digitali e applicazioni plastiche e, con il LT D1 e D2, le incisioni ondulate, a zig-zag, a reticolo e a pettine arrivano a coprire gran parte delle superfici esterne ${ }^{84}$ (Fig. 4). Gli scavi in vicolo S. Maria alla Porta e quelli lungo la linea metropolitana 3 avevano intercettato livelli

\footnotetext{
76 Anche ONGaro 1999, pp. 47-49, tavv. XV-XVI; Frontini - ONGARO 1996, figg. 27, nn. 8-9; 28, nn. 5-6, 11-12.

77 Per la pianura si veda, ad esempio, l'olletta ovoide decorata da impressioni digitali con trascinamento d'argilla dalla necropoli rurale di Urago d'Oglio (con datazione, su base stilistica, al La Tène B, cfr. DE MARINIS 2019, pp. 47-48, fig. 36).

78 BuOITE - ZAMbOni 2008; 2013; Morpurgo 2016.

${ }^{79}$ Per il quadro storico e archeologico si faccia riferimento a ORTALLI 2017, con bibliografia precedente.

$80 \mathrm{Al}$ Forte Urbano, nonostante le incertezze legate alle modalità di scavo (trincee esplorative di ridotte dimensioni e di difficile raccordo), i contesti (struttura 14, US 1207; probabili livelli di abbandono US 1211; US 1069) suggeriscono una cronologia non posteriore alla prima metà del IV sec. a.C. (BUOITE - ZAMBONI 2008; a meno di ipotizzare intrusioni di materiali di epoche successive, non suffragate però dalla presenza di altre classi). A Spina queste decorazioni provengono da fasi di abbandono e risistemazione dell'abitato (III-V), databili tra la seconda metà del IV e gli inizi del III sec. a.C. (BUOITE - ZAMBONi 2013). Anche a Marzabotto i materiali di fase "gallica" (tra le ceramiche decorate vi sono un'olla situliforme con decorazione a zig-zag e frammenti con impressioni a unghiate) non dovrebbero scendere oltre la metà del III sec. a.C. (Morpurgo 2016, tav. XIV). A Bologna-via Andrea Costa la fase III, con ceramica d'impasto decorata a incisioni, impressioni strumentali e digitali, si data tra la metà del IV e la metà del III sec. a.C. (TASSINARI 2010, pp. 90, 96, fig. 7).

81 ORTALLI 2008.

82 Ferrari - MENGoli 2005; Vitali 2014a, p. 298.

83 Per l'area insubre GRASSI 1995, pp. 85-88; GRASSI 2000. Lo stesso fenomeno si osserva nella pianura veronese: BIONDANI 2018, pp. 234-237.
}

${ }^{84}$ CASINI - FronTINI 2015, pp. 136-170. 
compresi tra la fine del II e l'età imperiale ${ }^{85}$, con forme e decorazioni analoghe, mentre sono residuali i frammenti da Piazza Sant'Ambrogio (olle e ciotole con incisioni ondulate e a pettine trascinato, impressioni strumentali a punti e triangoli, e impressioni digitali a unghiate con trascinamento $)^{86}$.

È soprattutto l'edizione delle necropoli di Oleggio e Dormelletto ${ }^{87}$ a permettere per questo periodo una scansione delle forme principali (Fig. 4): olle e ciotole-coperchio decorate, su cui compaiono durante il LT C1 incisioni lineari e a zig-zag poste sulla spalla dei vasi (Fig. 4). Nella fase LT C2 sembrano diffondersi maggiormente le impressioni strumentali (campiture di punti) e digitali, fino all'esplosione delle teorie esornative con il LT D1 (fitte campiture di incisioni e impressioni digitali, decorazioni plastiche a bugne e a punte di diamante), con un incremento quantitativo nella fase finale, LT D2 (70/60 - 30 a.C.). Il quadro trova sostanziali conferme in Lombardia in alcuni abitati minori, come Angera $^{88}$, e in insediamenti rustici di pianura e pedecollinari ${ }^{89}$.

La situazione nelle colonie romane fondate lungo le principali direttrici, la via Emilia e la Postumia, è di difficile valutazione dal momento che non sempre sono disponibili dati contestuali e quantitativi. A eccezione di Cremona, dove questa ceramica è molto abbondante anche nei livelli tardo-repubblicani e primo imperiali, e attribuita a un vivace mercato interno ${ }^{90}$.

A Piacenza è possibile che la ceramica d'impasto con decorazioni tipiche della fase tarda fosse prodotta localmente all'interno dei medesimi workshop che rifornivano la colonia di vernice nera e ceramiche comuni $^{91}$. I pochi esemplari da Parma o da Reggio Emilia ${ }^{92}$ (ollette decorate da incisioni lineari e impressioni), sono stati ricondotti ad attardamenti di substrato. A Modena alcuni frammenti provengono da strati connessi alle prime fasi della fondazione, precedenti gli impianti delle domus tardorepubblicane ${ }^{93}$. Nella bassa pianura padana, frammenti di pareti a unghiate con trascinamento d'argilla sono emersi dallo scavo della villa di Chiunsano (Ficarolo/Gaiba, RO) ${ }^{94}$.

\subsubsection{Calvatone/Bedriacum}

In questo quadro riveste particolare interesse il caso del vicus romano di Calvatone/Bedriacum dove numerose campagne di scavo - dirette negli ultimi due decenni da Maria Teresa Grassi - hanno restituito una cospicua quantità di ceramica d'impasto decorata. Un lotto (Fig. 5, 1-3) proviene dall'esplorazione del settore sud-ovest (Campo del Generale), in contesti databili tra l'ultimo quarto del II sec. a.C. e gli inizi del I sec. d.C. ${ }^{95}$.

\footnotetext{
85 ANGANUZZI et alii 1986; GUGLIELMETTI et alii 1991, pp. 169-170.

86 AIroldi 2015, fig. 1. È notizia recente il ritrovamento di contesti di fase lateniana nell'area dell'anfiteatro, inclusi numerosi frammenti di ceramica d'impasto decorata con incisioni e impressioni (CONSONNI et alii 2021, pp. 41-42). Una frequentazione in fase La Tène di quest'area meridionale era già indiziata da ritrovamenti precedenti presso S. Eustorgio (MILLS et alii 2007).

87 Poletti ECCLESIA 1999; RATTO 2009.

88 DOMANico 1995.

89 È il caso, ad esempio, di Rivanazzano Terme (PV) dove scavi recenti hanno documentato la presenza di frammenti decorati a impressioni strumentali (MAGGI et alii 2016, p. 627, fig. 5; BATTAGLIA - MAINO c.s.)

${ }^{90}$ CECCHINI - AIROLDI 2018.

${ }^{91}$ Marini Calvani 1990, p. 776; cfr. Grassi 1995, p. 88.

92 Podini - Losi 2019, p. 21 (F. Biondani); Biondani 2014, fig. 1, n. 7; MALnati 2013.

93 GuANDALini - BenASsi 2018, pp. 102-103.

${ }^{94}$ CORTI 2018, p. 206, fig. 1.1-4.

${ }^{2}$ Della PorTA - SFredda 1996, p. 152, tab. 19a-b.
} 
Nell'area della Domus del Labirinto vanno poi segnalati alcuni contesti chiusi (riempimenti di fosse, scarichi e livellamenti al di sotto di pavimenti) pertinenti alla prima fase dell'insediamento (fine II - fine I sec. a.C.), in cui vari frammenti d'impasto decorati (Fig. 5, 4-18) sono associati a forme datanti in vernice nera (quali ad esempio le patere Lamboglia 5 o le ciotole Lamboglia 28), lucerne biconiche dell'Esquilino, tegami a vernice rossa interna, e pareti sottili ${ }^{96}$.

Tra le poche forme riconoscibili, le olle ovoidi con orli estroflessi arrotondati e a breve tesa (Fig. 5, 1-3) rimandano all'ambito "cenomane" visto a Brescia (supra). Tra le decorazioni diffuse vi sono incisioni lineari (Fig. 5, 1), anche associate a impressioni ovali (Fig. 5, 2) o a unghiate (Fig. 5, 1). Sembrano godere di un discreto successo le impressioni strumentali triangolari (Fig. 5, 3, 12, 13, 15), in associazione con le semilune (Fig. 5, 16), e le "virgole" (Fig. 5, 14, forse 11), anche in questo caso in sintonia con quanto avviene nel capoluogo bresciano. Presenti anche le campiture a pettine (Fig. 5, 4), a impressioni ovali (Fig. 5, 8), e le impressioni digitali a unghiate (Fig. 5, 17).

\section{Interpretazioni: provenienza, influenze e usi}

Messo ordine, seppur in via preliminare, tra questioni terminologiche, tecniche e cronologiche, è il momento di affrontare problematiche di ambito culturale. Assodato che all'interno del record archeologico delle regioni in esame, Lombardia, Piemonte orientale, pianura veronese ed Emilia, si assiste a un cambiamento, più o meno sensibile, rispetto ai panorami ceramici precedenti (golasecchiano, veneto occidentale, etrusco-padano) ${ }^{97}$, quale spiegazione possiamo dare a tale fenomeno? Da dove proviene il gusto decorativo, sostanzialmente privo di antecedenti diretti locali, che abbiamo descritto nei paragrafi precedenti? Quando compare, in che ambiti si sviluppa, e fino a quando si protrae? E quali sono gli scenari interpretativi, in senso sociale, storico e culturale?

Per quanto riguarda le possibili aree di provenienza, in letteratura si oscilla sostanzialmente tra l'ipotesi di una diretta origine transalpina, dove incisioni, impressioni a strumento e digitali sono già attestate tra Hallstatt finale e La Tène iniziale ${ }^{98}$, e una mediazione del mondo ligure. Quest'ultima posizione, già prospettata da Filippo Gambari ${ }^{99}$, sostiene come alcuni prototipi francesi siano stati recepiti ed elaborati nella ceramica d'impasto ligure durante il V secolo a.C. Dalla Liguria costiera e interna si sarebbero poi diffusi in transpadana e in Emilia, a seguito delle invasioni storiche degli inizi del IV secolo a.C. Accolta in diversi studi ${ }^{100}$, e articolata soprattutto da Stefania Casini ${ }^{101}$, tale ipotesi si fonda sull'anteriorità dei materiali transalpini, in particolare nella regione del Rodano ${ }^{102}$, da dove avrebbero influenzato le produzioni liguri. Non si escludono tuttavia contatti diretti tra la pianura padana e le regioni transalpine: «non è improbabile che, all'indomani dell'invasione gallica, i modelli di ispirazione siano quelli francesi giunti senza la mediazione ligure» ${ }^{103}$.

\footnotetext{
${ }^{96}$ Grassi 2013, pp. 86, 90, 258, 261 (schede di G. Zenoni, L. Palmieri, S. Nava, D. Benedetti, F.A. Ossorio). In alcuni casi si tratta di materiali in giacitura secondaria, a seguito di azioni di pulizia, obliterazione o rifacimento di ambienti precedenti (es. US 9116, ivi, p. 211). I materiali editi, riportati in questa sede, sono solo un campione esiguo. È in corso una tesi di specializzazione, affidata a Filippo Molteni, dedicata allo studio sistematico di questa classe dagli scavi dell’Università di Milano a Calvatone.

${ }^{97}$ Vitali 2014a, p. 300.

98 Ad esempio Domanico 1995, p. 294, nota 69.

99 Gambari - Venturino Gambari 1988; GAMbari - Venturino GAMBARi 2004, p. 34. Cfr. De Marinis 2019 , p. 48.

100 BUOITE - ZAMBONI 2013; RAgAZZI - SOLANO 2014, p. 73.

101 CASINI - TIZZONI 2015, pp. 241-244

102 CASINI - TIZZONI 2015, p. 241; cfr. SEGUIER 2009.

103 CAsini - Tizzoni 2015, p. 244.
} 

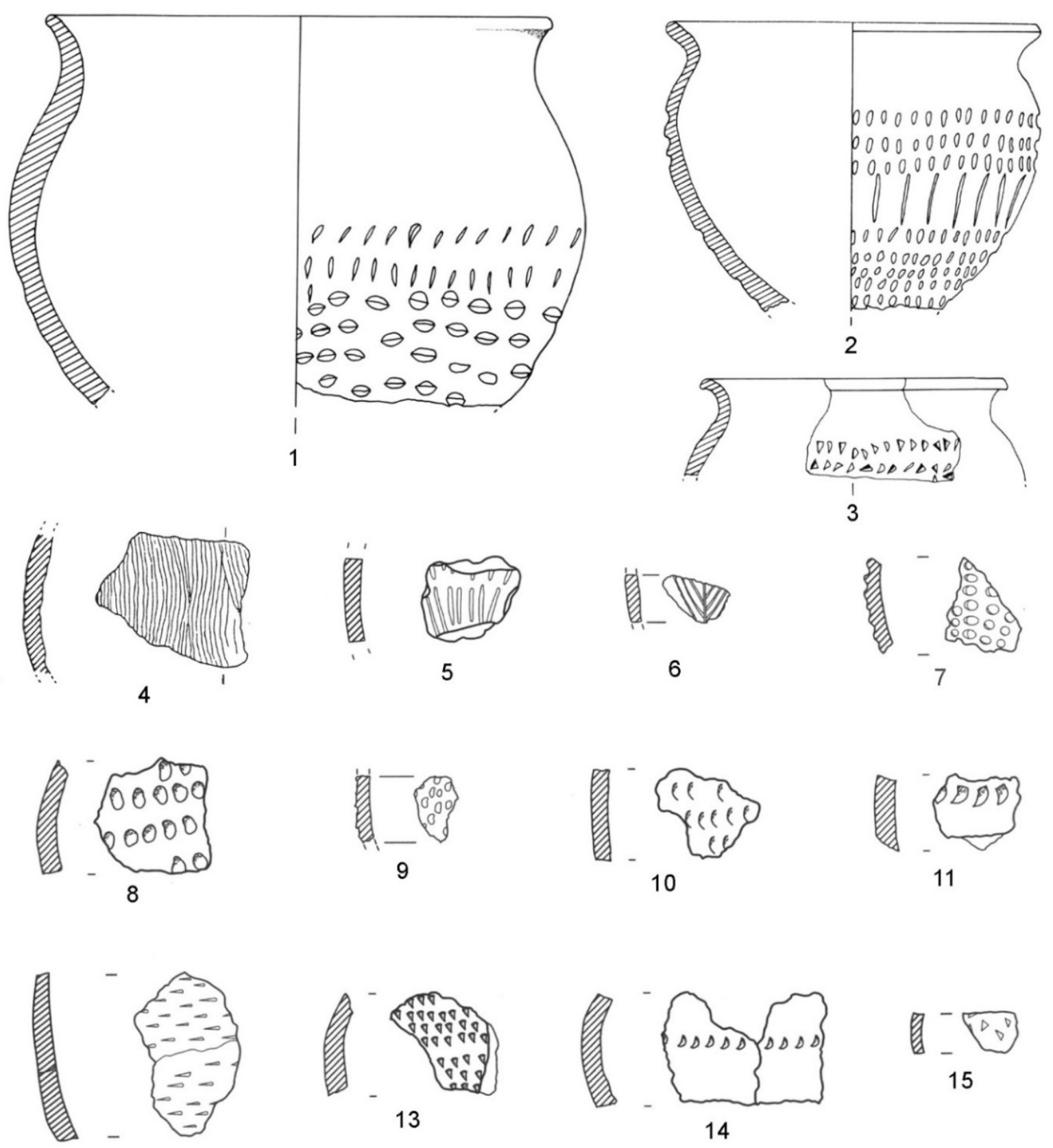

12

13

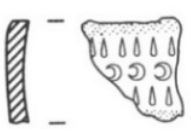

16
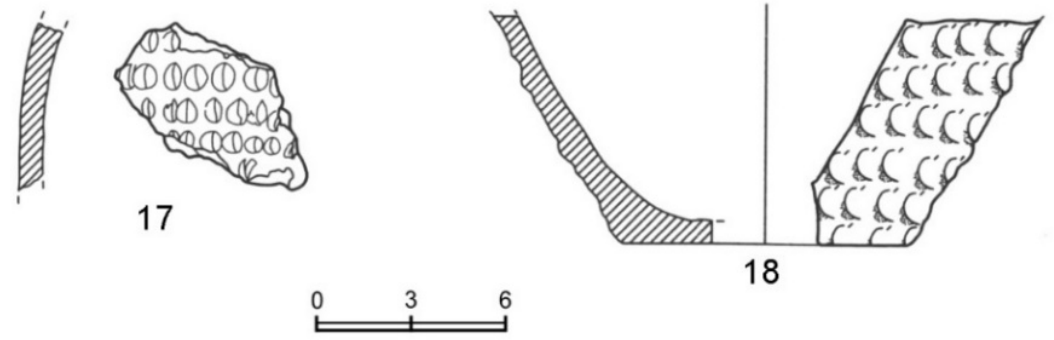

Fig. 5. Calvatone (CR), tavola esemplificativa dei frammenti decorati editi (1-3 da DELLA PORTA - SFREDDA 1996; 4-18 da GRASSI 2013). 
La questione è complessa, non solo perché manca ad oggi uno studio sistematico e comparativo di questa ceramica a livello sovra-regionale, ma anche perché si vanno a coinvolgere problemi teorici ampi: somiglianze formali tra periodi e aree differenti implicano automaticamente rapporti diretti o mediati? o sono frutto di coincidenze dettate, magari, dalla semplicità della tecnica impiegata e della moda? Si tratta in altre parole, ricorrendo a un tema cardine della biologia evoluzionistica ${ }^{104}$, di omologia o di analogia ${ }^{105}$ ?

Un'eventuale risposta presuppone ben altra scala di analisi e un maggior grado di approfondimento, soprattutto l'applicazione di analisi geostatistiche e tecnologiche ${ }^{106}$. A livello puramente descrittivo si può al momento suggerire quanto segue:

1) Alcune delle sintassi estranee al patrimonio precedente che, a partire dal La Tène B, si diffondono in territorio "insubre" (Lombardia, Piemonte orientale) come anche in Emilia e nella pianura veronese, sono già attestate in ambito ligure (Liguria, Piemonte meridionale, Appennino ligure/emiliano) tra VI e V secolo a.C. ${ }^{107}$ : si tratta in particolare di incisioni (lineari, ma soprattutto a zig-zag) concentrate sulla spalla delle forme chiuse, di alcune impressioni strumentali (circolari, triangolari), di impressioni digitali (unghiate, unghiate con trascinamento d'argilla, polpastrelli), e di alcune decorazioni plastiche (bugne, punte di diamante).

2) Alcune teorie sono comuni all'ambito ligure a quello transalpino: le incisioni lineari e a zig-zag, le impressioni digitali (unghiate e polpastrelli), le decorazioni plastiche (bugne, punte di diamante).

3) Altre decorazioni, sporadiche in contesti liguri, sono invece ben attestate in area transalpina già dal tardo Hallstatt/LT $\mathrm{A}^{108}$ : alcune incisioni (a onde, a spina di pesce, a reticolo) e le campiture a pettine; alcune impressioni strumentali, sia su file singole o doppie, ma soprattutto a fitte campiture (quadrangolari, rettangolari, virgole, semilune).

4) Alcune sintassi tipiche del mondo ligure e transalpino, al contempo, non hanno successo in pianura: ad esempio i cordoni, i cordoni digitati, e la decorazione convenzionalmente definita "stile di Kalenderberg" (supra).

Da queste osservazioni si ricaverebbe l'impressione del diffondersi in pianura padana, dagli inizi del IV sec. a.C., di influenze miste, provenienti sia dall'ambito ligure che da quello centro-europeo. Presupposti e motivazioni risiedono in un quadro complesso e articolato, che coinvolge altre classi e forme ceramiche (per non parlare degli oggetti d'ornamento, delle armi, della lingua e dei dati bioarcheologici), il che sorpassa evidentemente i limiti di questo lavoro. Ci limitiamo qui a osservare come tra seconda metà del IV e il III sec. a.C. compaiano in transpadana alcune forme in ceramica fine (olle piriformi, brocche a becco tubolare, alcune ceramiche dipinte, vasi "pre-trottola", ciotole "tipo

\footnotetext{
104 Gould 1986; Boniolo 2008, pp. 91-112.

105 Raffaele de Marinis nello specifico (trattando della decorazione “tipo Kalenderberg”) ammonisce: «Quando una tecnica o uno stile decorativo appaiono su vaste aree geografiche e in epoche differenti, non se ne può trarre alcuna conclusione su eventuali derivazioni o connessioni culturali» (DE MARINIS 2019, 49).

$106 \grave{\mathrm{E}}$ in corso un progetto di ricerca internazionale dedicato a queste ceramiche, pensato da chi scrive insieme a Jan Kysela (Praga), Richard Thér e Tomas Mangel (Hradec Králové), che coinvolga diverse regioni dell'Europa centrale e adotti un approccio comparativo, analitico (cfr. THÉR - MANGEL 2021) ed etnoarcheologico.

107 Ferrero et alii 2004; Marchiaro 2012; Mordeglia 2016. Ma che queste decorazioni siano il frutto di un'influenza transalpina $\mathrm{o}$, piuttosto, un'evoluzione a partire da antecedenti locali è una questione del tutto aperta.

108 SEGUIER 2009; BARDEL et alii 2017.
} 
Casalecchio" $)^{109}$ che, viste insieme all'impasto decorato, vanno a delineare una cultura ceramica che trae ispirazione da diverse regioni europee per formare però un aspetto locale originale.

Più che un mero marker di "latenizzazione", inteso semplicisticamente come processo di avvicendamento culturale, o persino di sostituzione etnica ${ }^{110}$, queste ceramiche sono piuttosto indicatori di adattamenti e sperimentazioni rispetto a un quadro economico e sociale mutato.

\subsection{Subalternità e resilienza}

Nei secoli successivi, II e I sec. a.C., durante l'espansione e la conquista romana, in alcune regioni, soprattutto nell'areale "insubre", la ceramica d'impasto decorata, come abbiamo visto, tende ad aumentare e ad articolarsi, con una persistenza anche allinterno di insediamenti pienamente romani (colonie, vici, campagne) e un picco statistico tra la fine del II sec. a.C. e l'età augustea. Pur scomparendo gradualmente nel corso della prima età imperiale, lo stile di questa ceramica sembra aver influenzato localmente produzioni di comune grezza ${ }^{111}$, di pareti sottili o di ceramica comune che imita le pareti sottili ${ }^{112}$.

Questa apparente contraddizione - l'aumento di una ceramica "indigena" durante il processo di romanizzazione, è già stata enfatizzata e affrontata in passato. Una prima ipotesi, avanzata da Ermanno Arslan, vedeva nella presenza di questo vasellame un segno di crisi delle comunità locali, di arretratezza e povertà, relegata alla sfera domestica, «indizio di un ultimo sviluppo autonomo di comunità celtiche ormai isolate e disorganizzate dalle linee di penetrazione romana nella regione» ${ }^{113}$. Non senza un ultimo slancio vitale, pur locale e periferico: «una facies culturale, quella delle trottole e delle bugnette, certamente povera, e di povera gente, ma non ancora priva di una certa vitalità, specialmente se consideriamo certe tendenze decorativistiche, spinte fino all'esasperazione» ${ }^{114}$.

Più neutra è invece l'idea che si tratti di semplici "ricordi di famiglia" inseriti nei corredi ${ }^{115}$, oppure di "conservatorismo" "116, cioè generiche sopravvivenze di tradizioni preromane, soprattutto nelle aree rurali dove vivevano «consumatori legati a tradizioni alimentari e a gusti decorativi non romani, siano essi di origine celtica oppure i liguri» ${ }^{117}$.

Queste suggestioni sono state riprese ed elaborate da Maria Teresa Grassi, la quale ha insistito piuttosto su forme di resistenza culturale ${ }^{118} \mathrm{o}$, diremmo oggi, di resilienza e negoziazione di una identità comune (percepita o inventata), basata su pratiche alimentari comuni e alternative a quelle romane. Un

\footnotetext{
109 Vitali 2014a; CASINI - TIZZONI 2015. La loro diffusione in Emilia è assai più limitata, sia in termini di forme che di quantità (VITALI 2014a).

${ }^{110}$ Da rigettare, o quantomeno da maneggiare con la massima cautela, sono infatti equazioni di matrice "storico-culturale" (un paradigma obsoleto ma notoriamente difficile da sradicare) che associano la presenza di ceramiche "lateniane" o "liguri" a individui o gruppi etnicamente connotati: ad esempio, «la presenza di questa ceramica al di fuori dei territori di origine permette di pensare allo spostamento di Liguri insieme ai gruppi di Galli invasori e al loro insediamento stabile» (CASINI TIZZONI 2015, p. 242).

111 Ad esempio, Rebaudo GreCo 1980; CORTESE 2003.

112 GRASSi 2000, p. 24; per il Comasco in particolare BUTTI RONCHETTI 2009-10, pp. 43-52, figg. 48-63.

113 ARSLAN 1971, p. 73. «Si tratta, a mio avviso, dell'ultima manifestazione vitale della ceramica celtica in transpadana, respinta, come era prevedibile date le premesse, in ambito autarchico famigliare» (ARSLAN 1977, p. 474).

114 ARSLAN 1971, p. 74.

115 BiNAGHi LEVA 1996, p. 266.

116 GuglielmetTI et alii 1991, p. 170; Croci 1996, p. 204.

117 BIONDANI 2014, p. 236.

118 GRASSI 1991, p. 122; GRASSI 1995, pp. 85-88; GRASSI 2000.
} 
«fenomeno per il quale si è supposta una valenza ideologica, vale a dire la sottolineatura di una identità nel momento in cui viene minacciata» ${ }^{19}$.

Una istanza di riconoscimento di tipo identitario ${ }^{120}$, dunque, che si sarebbe manifestata attraverso una produzione che «si colloca tuttavia certamente in un ambiente fortemente romanizzato apparendo in qualche modo incrementata proprio dal rapporto con il mondo romano» ${ }^{121}$.

\subsubsection{Ceramica indigena? L'esempio della Colonoware}

Il fenomeno della negoziazione e della difesa di un'identità culturale attraverso la reiterazione di determinate pratiche alimentari, specie da parte di comunità marginalizzate (in senso sociale oppure politico), ha naturalmente molti confronti etnografici e storici, soprattutto nell'ambito degli studi postcoloniali. Tra tutti, è suggestivo il confronto con la cosiddetta Colonoware (Fig. 6), un"“anomala" classe ceramica non tornita, cotta a basse temperature, caratteristica delle strutture abitate dagli schiavi africani, dai loro discendenti e da alcune comunità di nativi americani schiavizzati tra il tardo XVII fino alla metà del XIX secolo negli Stati del Sud, specialmente in South Carolina e Virginia.
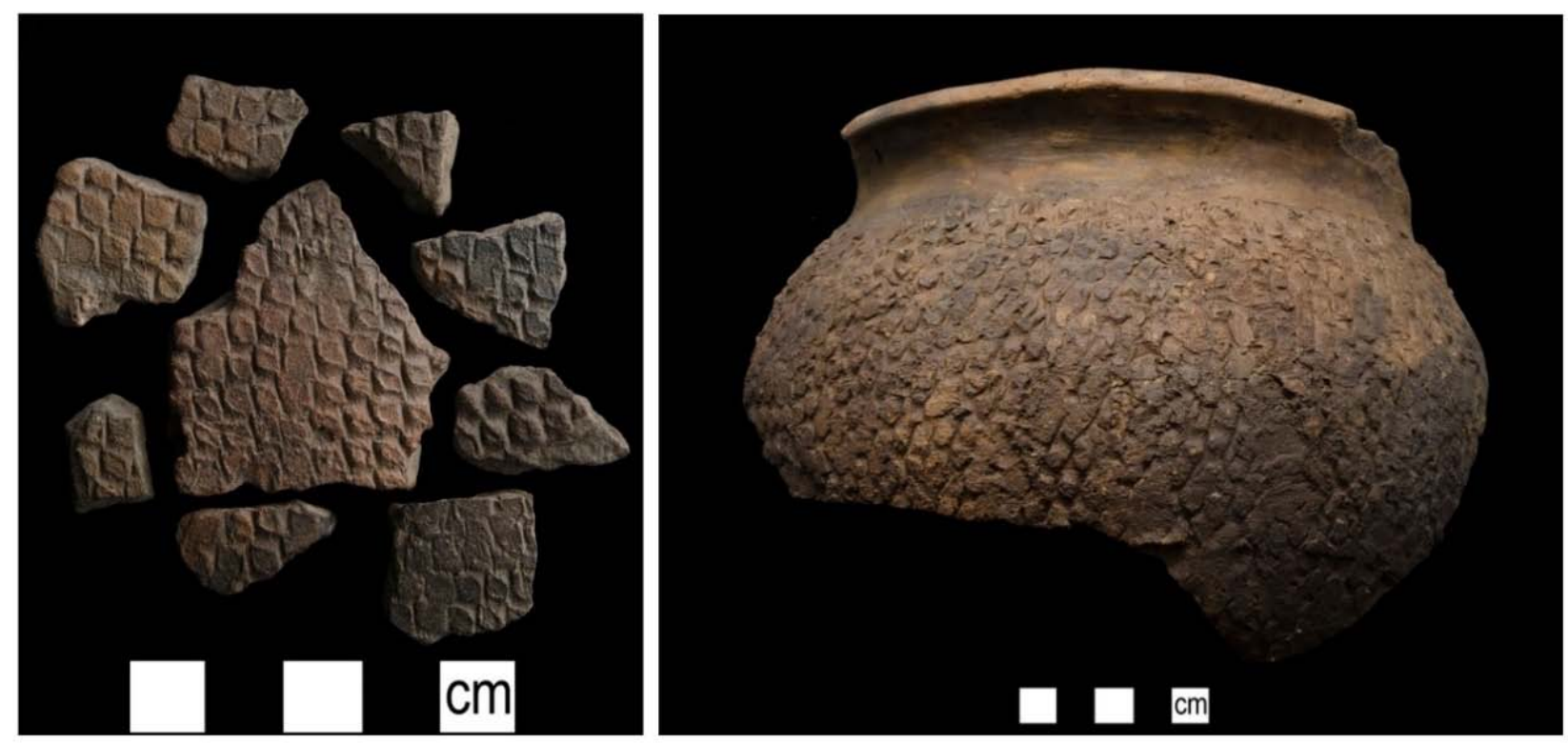

Fig. 6. Esempi di strip-rouletted Colonoware, dallo scavo di Drayton Hall (sinistra) e dal Horry County Museum (destra), South Carolina (da SATTES et alii 2020).

Inizialmente attribuita alle tribù native (Pamunky, Nottoway, Catawba), a partire dagli studi di James Deetz e Leland Ferguson ${ }^{122}$ si è ipotizzato come gli schiavi afroamericani continuassero a produrre, a livello domestico, olle e ciotole secondo tecniche, modelli e decorazioni derivati dall'Africa centro-occidentale ${ }^{123}$, pur con commistioni e ibridazioni con il vasellame nativo e quello di origine europea $^{124}$. Il ruolo di questa produzione sarebbe stato quello di reiterare costumi alimentari e pratiche

\footnotetext{
119 BiONDANi 2018, p. 239.

${ }^{120}$ Per le coordinate teoriche su questi temi si rimanda a ZAMBONI 2018, pp. 232-238.

121 SENA CHIESA 2014, p. 40.

122 Deetz 1996; Ferguson 1980; Ferguson 1991; Ferguson 1992.

123 SATTES et alii 2020.

124 COBB - DePratTer 2012; ChODORONEK 2013.
} 
di condivisione comunitarie del cibo alternativi (e in opposizione) alle mode importate e imposte dai coloni inglesi (ceramiche smaltate, porcellane e set di posate, da usare come servizi individuali).

Secondo questa lettura, l'uso attivo della tradizionale (anche se rielaborata) Colonoware avrebbe dunque rafforzato i legami sociali non gerarchici e un senso di appartenenza in seno alle comunità coloniali subalterne e marginali, che avrebbero sperimentato una forma di resilienza quotidiana nei confronti della cultura dominante bianca.

Altri studi hanno obbiettato che la questione è in realtà più complessa, e che un'interpretazione binaria della Colonoware focalizzata sulla componente etnica (come sola espressione della cultura degli schiavi afroamericani) sia riduttiva, se non fuorviante, poiché sottovaluta le origini multiple di queste tradizioni, l'interazione multiculturale, la pluralità delle componenti in gioco e l'eterogeneità dell'esperienza coloniale ${ }^{125}$.

\subsection{Chi usava questa ceramica, e per cosa?}

Cosa possiamo inferire dalla comparazione con altre classi ceramiche problematiche e "anomale", come la Colonoware? Innanzitutto, dobbiamo chiederci chi usasse questi vasi, nel quotidiano o durante le cerimonie funebri. Nonostante il racconto tradizionale (ma etico) delle fonti storiche ${ }^{126}$, possiamo dubitare che si siano verificate estese sostituzioni "etniche" di intere popolazioni a seguito dei fenomeni migratori del IV e del III sec. a.C., dal momento che sia l'archeologia che i (pochi) dati archeobiologici parlano a favore di un graduale cambiamento culturale, di un mescolamento e di un'integrazione tra popolazioni locali e gruppi di individui cresciuti in diverse regioni europee ${ }^{127}$.

Nella fase recenziore, dal LT C2 alla romanizzazione, neppure siamo di fronte a gruppi manifestatamente subalterni o marginali (da quello che possiamo intuire dalle strutture insediative e attraverso la lente dei corredi tombali), ma allo stesso tempo non si tratta di gruppi aristocratici di alto rango, generalmente poco visibili nell'areale e nel periodo in esame ${ }^{128}$. Anche eventuali distinzioni di genere non sono state finora prese in considerazione ${ }^{129}$, seppure ad un primo sguardo non appaiano significative in termini di distribuzione dei vasi decorati all'interno dei corredi.

Dobbiamo quindi pensare a classi sociali intermedie, composte da individui di provenienza eterogenea, che adottano un vasellame che non rappresentava un'unica tradizione, ma una combinazione di influenze stilistiche e manifatturiere diverse, soggette alla variabilità del mercato e dei gusti personali.

La destinazione d'uso rimane in sospeso a causa dell'assenza di dati analitici, gascromatografici: possiamo solo ipotizzare un legame con sostanze, usi e pratiche sociali che ne abbiano dettato il

\footnotetext{
125 COBB - DePratTer 2012, p. 449: «these distinctive ceramics were an outgrowth of colonial rivalries involving French, Spanish, and English empires, and interactions among African, Native American, and Euro-American peoples swept up in those rivalries».

126 VITALI 2014b.

127 Le analisi isotopiche condotte a Monte Bibele (SCHEERES et alii 2013) suggeriscono una bassa incidenza della mobilità umana $(19 \%)$, relativa soprattutto a individui maschili ma, sorprendentemente, non connessi alla classe dei guerrieri.

I dati di Monterenzio Vecchio (SORRENTINO et alii 2018) mostrano invece percentuali maggiori di individui non locali su base isotopica (58\%), ma anche in questo caso con un alto grado di eterogeneità: «isotope results cannot inform us whether allochtnous individuals are of possible Celtic origin, or if they rather belong to other, non-local Italian groups» (ivi, p. 18).

128 GRASSI 1995, p. 94.

${ }_{129}$ Vedi invece, per tonare al caso della Colonoware, il ruolo della componente femminile (COBB - DEPRATTER 2012, pp. 455456).
} 
successo, come ad esempio la preparazione e il consumo di bevande fermentate alternative al vino, oppure la cottura e il servizio di cibi e ricette particolari ${ }^{130}$. Che tali pratiche, e gli oggetti che attivamente le influenzavano ${ }^{131}$, avessero assunto un valore ideologico, come segno di appartenenza culturale e difesa di un'istanza identitaria, rimane un'ipotesi di lavoro accattivante ma probabilmente riduttiva, e necessita in ogni caso ulteriori approfondimenti.

Le prossime ricerche dovranno dunque indirizzarsi verso un'implementazione del dataset relativo a questa ceramica, anche a livello sovraregionale, che consenta elaborazioni geostatistiche e la definizione accurata di varianti locali. Sarà anche fondamentale impostare una ricerca tecnologica e microanalitica dei diversi stili e delle tecniche impiegate, insieme ad analisi interdisciplinari su tracce d'uso e resti organici, oltre a uno studio più raffinato di contesti, di cronologie e delle componenti sociali e di genere.

Lorenzo Zamboni lorenzo.zamboni@unimi.it

\section{Ringraziamenti}

Devo molti scambi di idee, suggerimenti e materiale bibliografico a colleghi e amici, tra i quali Marta Rapi, Marica Venturino, Diana Neri, Carla Buoite, Jan Kysela, Jacopo Ortalli e Paolo Rondini (ogni responsabilità di quanto scritto, incluse incomprensioni, imprecisioni e lacune, rimane naturalmente dell'autore).

\footnotetext{
130 GALLI 2005; BUTTI RONCHETTI 2009-10, pp. 50-52.

131 Volendo adottare la prospettiva del "material agency", o “material turn": VERSLUYS 2014; VAN OYEN 2017 (ma vedi FERNÁNDEZ-GÖTZ et alii 2020).
} 


\section{Abbreviazioni bibliografiche}

\section{AIROLDI 2015}

F. Airoldi, Ceramiche di età romana, in A.M. Fedeli - C. Pagani (a cura di), Il volto di una piazza. Indagini archeologiche per la realizzazione del parcheggio in Piazza Sant'Ambrogio a Milano, Milano 2015 (Archeologia preventiva e valorizzazione del territorio, 5), pp. 30-31.

Alle origini di Varese 2009

R.C. de Marinis - S. Mazza - M. Pizzo (a cura di), Alle origini di Varese e del suo territorio. Le collezioni del sistema archeologico provinciale, Roma 2009.

ANGANUZZI et alii 1986

A. Anganuzzi - A. Lavazza - M. Tizzoni, La ceramica comune, in CERESA MORI - TIZZONI 1986, pp. 173-243.

Anticbi silenzi 1996

Antichi silenzi. La necropoli romana di San Lorenzo di Parabiago, Legnano 1996.

\section{Arsago 1990}

Arsago. Nullus in Insubria pagus vetustior. Studi in memoria di Silvio Pozzi, Varese 1990.

\section{ARSLAN 1970-73}

E.A. Arslan, Per una più completa documentazione dell'età preromana nella provincia di Pavia, in "Rivista Archeologica dell'Antica Provincia e Diocesi di Como" 152-155 (1970-1973), pp. 475-483.

\section{ARSLAN 1971}

E.A. Arslan, Ritrovamenti preromani a Garlasco (località Madonna delle Bozzole), in Oblatio. Raccolta di studi di Antichità ed Arte in onore del Prof. Aristide Calderini, Como 1971, pp. 59-79.

\section{ARSLAN 1971-74}

E.A. Arslan, Spunti per lo studio del celtismo cisalpino, in "Rassegna di Studi del Civico Museo Archeologico di Milano" VII-X (197174), pp. 43

\section{ARSLAN 1972}

E.A. Arslan, Elementi per una classificazione tipologica della ceramica preromana a Garlasco, in M. Mirabella Roberti (a cura di), Archeologia e storia nella Lombardia padana. Atti del Convegno su Archeologia e storia nella Lombardia padana (Villa Monastero, 3-4 giugno 1969), Como 1972, pp. 123-155.

\section{ARSLAN 1977}

E.A. Arslan, Celti e Romani in Transpadana, in “Études Celtiques" 15.2 (1977), pp. 441-481.

BARDEL et alii 2017

D. Bardel - M. Saurel - L. Augier - H. Delneff S. Desenne - F. Di Napoli - R. Labeaune - Ch. Maitay, Géographie culturelle de la céramique décorée entre le VIe et le IVe siècle avant notre ère dans le bassin Parisien et ses marges, in S. Marion - S. Deffressigne - J. Kaurin - G. Bataille (éd.), Production et proto-industrialisation aux âges du Fer. Perspectives sociales et environnementales, Actes du $39^{\mathrm{e}}$ colloque international de l'AFEAF (Nancy, 14-17 mai 2015), Bordeaux 2017 (Collection Mémoires, 47), pp. 187-230.

\section{BATTAGLIA - MAINO}

M. Battaglia - E.V. Maino, Rivanazzano Terme: il sito plurifase di Cascina Isola Felice, in S. Maggi M. Battaglia - L. Zamboni (a cura di), Edifici rustici romani tra pianura e Appennino. Stato della ricerca, Firenze (Flos Italiae. Documenti di archeologia della Cisalpina Romana), in stampa.

BINAGHI LEVA 1996

M.A. Binaghi Leva, I dati emersi dalle indagini, in Antichi silenæi 1996, pp. 264-270.

\section{BIONDANI 2013}

F. Biondani, Le produqioni ceramiche nella pianura veronese fra il VT e il II sec. a.C.: aspetti cronologici, tipologici e distributivi, tesi di Dottorato, a.a. 20122013, XXV ciclo, Università di Padova. 
BIONDANI 2014

F. Biondani, Identità culturale celtica ed identità culturale romana nella Cisalpina di II-I sec. a.C. Il dato della ceramica, in "Rei Cretariae Romanae Factorum Acta" 43 (2014), pp. 233-240.

\section{BIONDANI 2018}

F. Biondani, Fra Celti Cenomani e Romani. La ceramica nel territorio veronese dal III sec. a.C. all'età augustea: novità e persistenze, in "Rei Cretariae Romanae Factorum Acta" 45 (2018), pp. 229240.

\section{BONIOLO 2008}

G. Boniolo (a cura di), Filosofia e scienze della vita. Un'analisi dei fondamenti della biologia e della biomedica, Milano 2008.

\section{Brogiolo 1984}

G.P. Brogiolo (a cura di), Archeologia urbana in Lombardia. Valutazione dei depositi archeologici e inventario dei vincoli, Modena 1984.

\section{BUOITE - ZAMBONI 2008}

C. Buoite - L. Zamboni, I materiali, in D. Neri L. Malnati (a cura di), Gli scavi di Castelfranco Emilia presso il Forte Urbano. Un abitato etrusco alla vigilia delle invasioni celtiche, Firenze 2008, pp. 57172.

BUOITE - ZAMBONI 2012

C. Buoite - L. Zamboni, Ceramiche di tradirione La Tène nella pianura modenese, (https://www.academia.edu/41189447/BUOI TE_ZAMBONI_2012_Ceramiche di tradizio ne $\mathrm{La} \mathrm{T} \% \mathrm{C} 3 \% \mathrm{~A} 8$ ne nella pianura modenes e).

BUOITE - ZAMBONI 2013

C. Buoite - L. Zamboni, Ceramica d'impasto di tradiz̧ione La Tène, in C. Cornelio Cassai - L. Malnati - S. Giannini (a cura di), Spina. Scavi nell'abitato della città etrusca 2007-2009, Firenze 2013, 133-135.

BUTTI RONCHETTI 2009-10

F. Butti Ronchetti, Sulle tracce dei Comenses, nell'incontro con $i$ Romani, in "Rivista Archeologica dell'Antica Provincia e Diocesi di Como", 191-192 (2009-2010), pp. 7-52.
CALANDRA et alii 1998

E. Calandra - A. Failla - C. Melley - C. Scalari, Problemi di tecnologia e di produzione: la ceramica grezza dell'età della romanizzazione a Lomello, in Proceedings of the XIII International Congress of prehistoric and protohistoric sciences (Forlì, 8-14 september 1996), 5, Forlì 1998, pp. 77-82.

\section{CASINI - TIZZONI 2015}

S. Casini - M. Tizzoni, Via Moneta: analisi culturale delle fasi preromane / La produzione ceramica preromana: analisi delle forme, in A. Ceresa Mori (a cura di), Lo scavo di via Moneta a Milano (1986-1991). Protostoria e romanizzazione, "Notizie Archeologiche Bergomensi" 23 (2015), pp. 69-266.

CATTANEO 2003

R. Cattaneo, Osservazioni sulla ceramica comune tardo-celtica degli scavi di Laus Pompeia, "Rivista archeologica dell'antica provincia e diocesi di Como" 185 (2003), pp. 185-219.

CECCHINI - AIROLDI 2018

N. Cecchini, F. Airoldi, Ceramiche comuni da fuoco, in L. Arslan Pitcher, con E. Arslan - P. Blockley - M. Volonté (a cura di), Amoenissimis...aedificiis. Gli scavi di piazza Marconi a Cremona. II, I materiali, Mantova 2018, pp. 89-128.

Ceresa Mori - Tizzoni 1986

A. Ceresa Mori - M. Tizzoni (a cura di), Santa Maria alla Porta: uno scavo nel centro storico di Milano, "Studi archeologici" 5 (1986).

CHODORONEK 2013

M. Chodoronek, Colonoware and Culture: The Changing Interpretation of 17th Century Ceramic Traditions in the South Eastern United States: An Overview of Current Thought and History, in "Nebraska Anthropologist" 183 (2013), pp. 57-71.

COBB - DEPRATTER 2012

C.R. Cobb - C.B. DePratter, Multisited Research on Colonowares and the Paradox of Globalization, in "American Anthropologist", 2012, n.s. 114.3, pp. 446-461.

COLLIS 2003

J. Collis, The Celts. Origins, myths and inventions, Stroud 2003.

CONSONNI et alii 2021

D. Consonni - A.M. Fedeli - A. Ranaldi - F. Roncoroni, Milano. Contributi allo studio di Milano 
in età protostorica e romana nell'area dell'anfiteatro. Prime notizie degli scavi in corso (2019-2020), in "Notizie degli Scavi di Antichità" n.s. I, vol. I (2021), pp. 3-46.

\section{CORTESE 2003}

C. Cortese, Le ceramiche comuni. Forme $e$ produrioni tra l'età angustea e il III secolo d.C., in S. Lusuardi Siena - M.P. Rossignani (a cura di), Ricerche archeologiche nei cortili dell'Università Cattolica. Dall'Antichità al Medioevo. Aspetti insediativi e manufatti, Milano 2003, pp. 67-83.

\section{CORTI 2018}

C. Corti, Traffici nel Delta e lungo il corso del Po. Alcune osservazioni sulla circolazione di ceramiche ad impasto grez:o e anfore, in M. Cesarano - M.C. Vallicelli - L. Zamboni (a cura di), Antichi Romani e romanità nelle terre del Delta del Po. Nuovi studi e prospettive di ricerca, Bologna 2018, pp. 205-215.

\section{CROCI 1996}

A. Croci, La ceramica d'uso domestico, in Antichi silenæi 1996, pp. 201-208.

\section{CROTTI 2019}

M. Crotti, La ceramica di tradirione preromana, in S. Campagnari - F. Foroni - D. Neri (a cura di), Una sosta lungo la via Emilia, tra selve e paludi. La mansio di Forum Gallorum a Castelfranco Emilia, Forlimpopoli 2019, pp. 85-92.

DEETZ 1996

L. Deetz, In Small Things Forgotten: An Archaeology of Early American Life, New York 1996 [1977].

DELLA PORTA et alii 1998

C. Della Porta - N. Sfredda - G. Tassinari, Ceramiche comuni, in G. Olcese (a cura di), Ceramiche in Lombardia tra II secolo a.C. e VII secolo d.C. Raccolta dei dati editi, Mantova 1998 (Documenti di Archeologia, 16), pp. 133-229.

DELLA PORTA - SFREDDA 1996

C. Della Porta - N. Sfredda, La ceramica comune, in PASSi PITCher 1996, pp. 133-186.

DE MARINIS 1977

R.C. de Marinis, The La Tène Culture of the Cisalpine Gauls, in M. Guštin (ed.), Keltske Študije, Brežice 1977, pp. 23-50.
DE MARINIS 1986

R.C. de Marinis, L'età gallica in Lombardia (IV-I secolo a.C.): risultati delle ultime ricerche e problemi aperti, in Archeologia in Lombardia, Atti del $2^{\circ}$ Convegno archeologico regionale (Como, 13-15 aprile 1984), Como 1986, pp. 93-173.

DE MARINIS 2019

R.C. de Marinis, Sui riti funerari della cultura di Golasecca, in "IpoTESI di Preistoria" 11 (2019), pp. 1-56.

\section{DOMANICO 1995}

L. Domanico, Ceramica decorata del periodo tardo La Tène, in G. Sena Chiesa - M.P. Lavizzari Pedrazzini (a cura di), Angera Romana. Scavi nell'abitato 1980-1986, Roma 1995, pp. 75-77, 283-299.

\section{FERGUSON 1980}

L. Ferguson, Looking for the "Afro" in ColonoIndian Pottery, in R.L. Schuyler (ed.), Archaeological Perspectives on Ethnicity in America. Afro-American and Asian American Culture History, New York 1980, pp. 14-28.

FERGUSON 1991

L. Ferguson, Struggling with Pots in Colonial South Carolina, in R. McGuire - R. Paynter (eds.), The Archaeology of Inequality, Oxford 1991, pp. 2839.

\section{FERGUSON 1992}

L. Ferguson, Uncommon ground: archaeology and early African America, 1650-1800, Washington DC 1992.

FERNÁNDEZ-GÖTZ et alii 2020

M. Fernández-Götz - D. Maschek - N. Roymans, The dark side of the Empire: Roman expansionism between object agency and predatory regime, in "Antiquity" 94 (378) (2020), pp. 16301639.

FERRARI - MENGOLI 2005

S. Ferrari - D. Mengoli, I materiali di età celtica dalla struttura 2 di Casalecchio di Reno (BO), zona "A", in D. Vitali (a cura di), Studi sulla media e tarda età del Ferro nell'Italia settentrionale, Bologna 2005 (Studi e Scavi, n.s. 12), pp. 15-148. 
FERRERO 2007

L. Ferrero, "Dertona, città dei Liguri". I materiali della seconda età del Ferro e di tradiz̨ione preromana, in A. Crosetto - M. Venturino Gambari (a cura di), Onde nulla si perda. La collezione archeologica di Cesare Di Negro-Carpani, Alessandria 2007, pp. 135-148.

FERRERO et alii 2004

L. Ferrero - M. Giaretti - S. Padovan, Gli abitati della Liguria interna: la ceramica domestica, in Ligures Celeberrimi 2004, pp. 51-70.

\section{FORTUNATI ZUCCÀLA 1979}

M. Fortunati Zuccàla, 1979, Gropello Cairoli (Pavia). La necropoli romana, in "Notizie degli Scavi di Antichità" XXXIII (1979), pp. 5-87.

FRONTINI - ONGARO 1996

P. Frontini - G. Ongaro, Brescia tra l'età del Bronzo e l'età gallica, in Carta archeologica della Lombardia: 5. Brescia. La città, Modena 1996, pp. 23-71.

\section{GALLI 2005}

M. Galli, Vasellame domestico e Lebenswelt: nascita della cultura urbana nella colonia romana di Ariminum, in P. Zanker - R. Neudecker (a cura di), Lebenswelten, Bilder und Räume in der römischen Stadt der Kaiserzeit, Wiesbaden 2005, pp. 153173.

\section{GAMBARI - VENTURINO GAMBARI 1988}

F.M. Gambari - M. Venturino Gambari, Contributi per una definizione archeologica della seconda età del Ferro nella Liguria interna, in "Rivista di Studi Liguri", LIII, 1988, pp. 77150.

GAMBARI - VENTURINO GAMBARI 2004

F.M. Gambari - M. Venturino Gambari, La medio-tarda età del Ferro (V-II secolo a.C.) nella Liguria interna, in Ligures Celeberrimi 2004, pp. 29-48.

GIORGI et alii 2009-10

M. Giorgi - S. Martinelli - F. Butti Ronchetti, La necropoli romana di Rovello Porro (CO), in "Rivista Archeologica dell'Antica Provincia e
Diocesi di Como", 191-192 (2009-2010), pp. 53-288.

GOULD 1986

S.J. Gould, Evolution and the Triumph of Homology, or Why History Matters, in "American Scientist" 74 (1986), pp. 60-69

GRASSI 1991a

M.T. Grassi, I Celti in Italia, Milano 1991 (Biblioteca di Archeologia, 16).

GRASSI 1991b

M.T. Grassi, Insubri e Romani: un modello di integrazione, in "Sibrium" 21 (1990-91), pp. 279291.

GRASSI 1991c

M.T. Grassi, Ricerche di superficie condotte a Calvatone negli anni 1986/87, in G.M. Facchini (a cura di), Calvatone Romana. Studi e ricerche preliminari, Milano 1991 (Quaderni di Acme, 13), pp. 101-130.

GRASSI 1995

M.T. Grassi, La romanizzazione degli Insubri. Celti $e$ Romani in Transpadana attraverso la documentazione storica e archeologica, Milano 1995 (Collana di Studi di Archeologia Lombarda, 1).

GRASSI 2000

M.T. Grassi, Una produzione fittile di tradizione celtica: la diffusione della ceramica decorata a Milano e nel suo territorio, in Milano tra l'età repubblicana e l'età angustea, Atti del Convegno di studi (Milano, 26-27 marzo 1999), Milano 2000, pp. 19-29.

GRASSI 2008

M.T. Grassi, La ceramica a vernice nera di Calvatone-Bedriacum, Firenze 2008 (Flos Italiae. Documenti di archeologia della Cisalpina Romana, 7).

GRASSI 2013

M.T. Grassi (a cura di), Calvatone-Bedriacum. I nuovi scavi nell'area della Domus del Labirinto (2001-2006), "Postumia" 24, 3 (2013). 
GRASSI - MANGANI 2012

B. Grassi - C. Mangani, Catalogo dei corredi. Alcune riflessioni sulla necropoli di Urago d'Oglio, in F. Rossi - S. Solano (a cura di), Terre di confine. Una necropoli dell'età del Ferro a Urago d'Oglio, Milano 2012, pp. 21-39.

\section{GRAUE 1974}

G. Graue, Die Graberfelder von Ornavasso, Hamburg 1974.

GROSSETTI 2002

E. Grossetti, L'abitato di Pianello nel quadro del popolamento romano della Val Tidone, Piacenza 2002.

GUANDALINI - BENASSI 2018

F. Guandalini - F. Benassi, La Domus di via Università (ex cinema Capitol), in Mutina splendidissima 2018, pp. 102-109.

GUGLIELMETTI et alii 1991

A. Guglielmetti - L. Lecca Bishop - L. Ragazzi, Ceramica comune, in D. Caporusso (a cura di), Scavi MM3. Ricerche di archeologia urbana a Milano durante la costruzione della linea 3 della Metropolitana 1982-1990, Milano 1991, pp. 133257.

GUGLIELMETTI - SOLANO 2010

A. Guglielmetti - S. Solano, La ceramica comune del primo santuario romano tra età giulio-claudia e prima età flavia, in F. Rossi (a cura di), Il santuario di Minerva. Un luogo di culto a Breno tra protostoria ed età romana, Milano 2010, pp. 245-259.

\section{KYSELA 2020}

J. Kysela, Things and Thoughts. Central Europe and the Mediterranean in the $4^{\text {th }}-1^{\text {st }}$ centuries $B C$, Prague 2020 (Studia Hercynia, monographs 1).

\section{LEJARS 2006}

T. Lejars, Les Celtes d'Italie, in M. Szabò (dir.), Celtes et Gaulois, l'archéologie face à l'histoire, 3 : les civilisés et les Barbares (du V au II siècle avant J.C.), Actes du colloque (Budapest, 17-18 juin 2005), Glux-en-Glenne 2006, pp. 77-96.

\section{LEVI 2010}

S. Levi, Dal coccio al vasaio. Manifattura, tecnologia e classificazione della ceramica, Bologna 2010.
Ligures Celeberrimi 2004

M. Venturino Gambari - D. Gandolfi (a cura di), Ligures Celeberrimi. La Liguria interna nella seconda età del ferro, Atti del Convegno Internazionale (Mondovì 2002), Bordighera 2004.

\section{LOCATELLI 2018}

D. Locatelli, Prima di Roma: il periodo delle invasioni galliche, in Mutina splendidissima 2018, pp. 26-29.

MAGGI et alii 2016

S. Maggi - M. Battaglia - B. Peverelli M.T.A. Robino 2016, Rivanazzano Terme (Pv), località Cascina Pizzone. Prima campagna di ricognizione superficiale, in "Athenaeum" 104, II, pp. 621-630.

MALNATI 2013

L. Malnati, I precedenti: la fase gallica, in D. Locatelli - L. Malnati - D. Maras (a cura di), Storie della prima Parma. Etruschi, Galli, Romani: le origini della città alla luce delle nuove scoperte archeologiche, Catalogo della mostra (Parma, 12 gennaio - 2 giugno 2013), Roma 2013, pp. 5961.

MARCHIARO 2012

S. Marchiaro, Cronotipologia della ceramica d'impasto dell'abitato protostorico di Breolungi (Mondovi, Cuneo), in "Quaderni della Soprintendenza archeologica del Piemonte" 27 (2012), pp. 43-59.

\section{MARINI CALVANI 1990}

M. Marini Calvani, La città, in Storia di Piacenza. 1. Dalle origini all'anno Mille, 2, Piacenza 1990, pp. 774-786.

METE-RIDOLFI 2014

G. Mete - G. Ridolfi, Gli insediamenti rurali di età romana, in N. Cecchini (a cura di), Progresso e passato. Nuovi dati sul Cremonese in età antica dagli scavi del Metanodotto Snam Cremona-Sergnano, Milano 2014 (Archeologia preventiva e valorizzazione del territorio, 4), pp. 39-54.

MILLS et alii 2007

J. Mills - L. Ragazzi - S. Casini - M. Tizzoni, Milano. Chiostri di S. Eustorgio, in "Notiziario 
Soprintendenza Archeologica Lombardia" 2007, pp. 135-139.

\section{MORDEGLIA 2016}

L. Mordeglia, Rozza ceramica d'impasto. La ceramica ligure nell'età del Ferro, Roma 2016 (Officina Etruscologia 12).

MORPURGO 2016

G. Morpurgo, La fase tarda di Marzabotto, in E. Govi (a cura di), Il mondo etrusco e il mondo italico di ambito settentrionale prima dellimpatto con Roma (IV-II secolo a.C.), Atti del Convegno (Bologna, 28 febbraio - 1 marzo 2013), Pisa-Roma 2016, pp. 127-169.

\section{Mutina splendidissima 2018}

L. Malnati - S. Pellegrini - F. Piccinini - C. Stefani (a cura di), Mutina splendidissima. La città romana e la sua eredità, Roma 2018.

\section{Negroni CATACCHIO 1975}

N. Negroni Catacchio, Le fasi finali della civiltà di Golasecca nell'ambito degli aspetti culturali della Valpadana fino alla romanizzazione, in Popoli $e$ civiltà dell'Italia antica, IV, Roma 1975, pp. 329356.

\section{Negroni Catacchio 1982}

N. Negroni Catacchio, Scavi a Pianvalle (Como): $i$ rinvenimenti di epoca La Tène, in Studi in Onore di Ferrante Rittatore Vonwiller, Como 1982, I, pp. 315-353.

\section{ONGARO 1999}

G. Ongaro, Il materiale preromano, in G.P. Brogiolo (a cura di), S. Giulia di Brescia, gli scavi dal 1980 al 1992. Reperti preromani, romani e alto medievali, Firenze 1999, pp. 25-54.

\section{ORTALLI 2008}

J. Ortalli, L'insediamento celtico di Casalecchio di Reno (Bologna), in D. Vitali - S. Verger (a cura di), Tra mondo celtico e mondo italico. La necropoli di Monte Bibele, Atti della Tavola Rotonda (Roma 1997), Bologna 2008, pp. 299-322.

\section{ORTALLI 2017}

J. Ortalli, Romanizzazione e persistenze celtiche a sud del Po (III-I secolo a.C.), in Piana Agostinet'ti 2017, pp. 317-352.
PANCALDI 2010

P. Pancaldi, Spilamberto, Cava Ponte del Rio, Via Macchioni. Insediamento rustico e sepolcreti di età romana e altomedievale, in D. Labate (a cura di), Notizie degli scavi e delle ricerche archeologiche nel Modenese (2008), "Atti e Memorie della Deputazione di Storia Patria per le Antiche Provincie Modenesi" s. XI, XXXII (2010), pp. 343-346.

PASSI PITCHER 1996

L. Passi Pitcher (a cura di), Bedriacum. Ricerche archeologiche a Calvatone. 1.2, Il Campo del Generale: i materiali del Saggio 6, Milano 1996.

\section{PiAnA AgostinetTi 1972}

P. Piana Agostinetti, Documenti per la protostoria della Val d'Ossola. San Bernardo d'Ornavasso e le altre necropoli preromane, Milano 1972.

\section{PiAnA AgostinetTI 2017}

P. Piana Agostinetti (a cura di), Celti d'Italia. I Celti nell'età di La Tène a sud delle Alpi, Atti del Convegno Internazionale (Roma, 16-17 dicembre 2010), Roma 2017.

PODINI - LOSI 2019

M. Podini - A. Losi (a cura di), La città che si rinnova. Gli scavi di Palazzo Busetti e Piazza della Vittoria a Reggio Emilia, Parma 2019.

\section{POLETTI ECCLESIA 1999}

E. Poletti Ecclesia, Due tradizioni produttive per le forme da cucina e da tavola. La ceramica comune, in SPAGNOLO GARZOLI 1999, pp. 303-320.

Poggiani KeLLER 2006

R. Poggiani Keller (a cura di), L'oppidum degli Orobi a Parre (BG), Milano 2006.

PREDIERI - SFRECOLA 1996

G. Predieri - S. Sfrecola, Analisi mineropetrografiche, in PASSI PITCHER 1996, pp. 209213.

RAGAZZI - FRONTORI 2018

L. Ragazzi - I. Frontori, Ceramica comune da mensa, da dispensa e di uso vario, in L. Arslan Pitcher, con E. Arslan - P. Blockley - M. Volonté (a cura di), Amoenissimis...aedificiis. Gli scavi di piazza Marconi a Cremona. II, I materiali, Mantova 2018, pp. 29-88. 
RAGAZZI - SOLANO 2014

L. Ragazzi - S. Solano, La ceramica comune dalla fase etrusco-padana alla romanizzazione. Considerazioni sul settore 4, in F. Rossi (a cura di), Un luogo per gli dè̀. L'area del Capitolium a Brescia, Firenze 2014, pp. 55-121.

\section{RAPI 2009a}

M. Rapi, La seconda età del Ferro nell'area di Como e dintorni, materiali La Tène nelle collezioni del Civico Museo Archeologico P. Giovio, Como 2009 (Archeologia dell'Italia settentrionale, 11).

\section{RAPI 2009b}

M. Rapi, L'orizzonte tardo La Tène della necropoli di S. Ambrogio ad Arsago, in Alle origini di Varese 2009, pp. 537-541.

RAPI 2009c

M. Rapi, Materiali tardo La Tène dalla collezione Visconti in Somma Lombardo, in Alle origini di Varese 2009, pp. 645-648.

\section{RAPI 2009d}

M. Rapi, Tombe galliche da Ca' di Ass, Gallarate, in Alle origini di Varese 2009, pp. 690-692.

RAPI 2009e

M. Rapi, La tomba gallica di Piazza Ponti di Gallarate, in Alle origini di Varese 2009, pp. 693698.

\section{RATTO 2009}

S. Ratto, Il quotidiano oltre la morte. La ceramica comune, in SPAGNOLO GARZOLI 2009, pp. 179192.

\section{REBAUDO GRECO 1980}

G. Rebaudo Greco, La decorazione della ceramica comune di Caselette, in Studi di Archeologia dedicati a Pietro Baroncelli, Torino 1980, pp. 135-149.

\section{REMOTTI 2019}

F. Remotti, Somiglianze. Una via per la convivenza, Roma-Bari 2019.

\section{RossI 2002}

F. Rossi (a cura di), Urago d'Oglio. Ricerche archeologiche al Castellaro. Prime indagini sistematiche (1996-97), Milano 2002.
SALZANI 1995

L. Salzani, La necropoli gallica di Valeggio sul Mincio, Mantova 1995 (Documenti di archeologia, 5).

\section{SALZANI 1996}

L. Salzani, La necropoli gallica e romana di S. Maria di Zevio (Verona), Mantova 1996 (Documenti di archeologia, 9).

SALZANI 1998

L. Salzani, La necropoli gallica di Casalandri a Isola Rizza (Verona), Mantova 1998 (Documenti di archeologia, 14).

SATTES et alii 2020

C.A.H. Sattes - J.B. Marcoux - S.E. Platt - M. Zierden - R. W. Anthony, Preliminary Identification of African-Style Rouletted Colonoware in the Colonial South Carolina Lowcountry, in "Journal of African Diaspora Archaeology and Heritage", https://doi.org/10.1080/21619441.2020.1840 $\underline{837}$.

SCHEERES et alii 2013

M. Scheeres - C. Knipper - M. Hauschild - M. Schönfelder - W. Siebel - D. Vitali - C. Pare K.W. Alt, Evidence for "Celtic migrations"? Strontium isotope analysis at the early La Tène (LT B) cemeteries of Nebringen (Germany) and Monte Bibele (Italy), in "Journal of Archaeological Science” 40 (2013), pp. 3614-3625.

\section{SCHEFFLER 2018}

S.U. Scheffler, Nothing has happened? The integration of the Lomellina into the Roman empire, $\mathrm{PhD}$ thesis, University of Leicester.

SCHINDLER-KAUDELKA - BIONDANI 2018 E. Schindler-Kaudelka - F. Biondani, Stranieri nella ceramica grezza del Magdalensberg. Tracce di commercializzazione precoce oppure beni personali dei migranti?, in M. Cavalieri - C. Boschetti (a cura di), Multa per aequora. Il polisemico significato della moderna ricerca archeologica. Omaggio a Sara Santoro, Louvain 2018, pp. 241-261.

SEGUIER 2009

J. M. Séguier, La céramique domestique de l'espace culturel sénonais du milieu du Ve au milieu du IIIe s. 
av. J.-C. dans son contexte du centre-est de la France: corpus, faciès et évolution des assemblages du confluent Seine - Yonne, de la Bassée et de la vallée de l'Yonne, in "Revue archéologique de l'Est" 58 (2009), pp. $57-132$.

\section{SENA CHIESA 2014}

G. Sena Chiesa, Gli asparagi di Cesare. Riflessioni su cinquant'anni di archeologia cisalpina, in G. Sena Chiesa, Gli asparagi di Cesare. Studi sulla Cisalpina Romana, Firenze 2014, pp. 9-22.

\section{SIMONE 1986}

L. Simone, Necropoli gallica di Somma Lombardo, in "Sibrium" XVIII (1985-86), pp. 99-113.

\section{SIMONE ZOPFI 2008}

L. Simone Zopfi, Albairate (MI): tomba di guerriero degli inizi del III secolo a.C., in "FastiOnLine" www.fastionline.org/docs/FOLDER-it2008-106.pdf.

SIMONE ZOPFI et alii 2009

L. Simone Zopfi - R. Bitelli - A. Pittari - A. Simoncelli, Resti di età romana, tardoromana e moderna lungo il tracciato del metanodotto SettalaRodano (MI), in "FastiOnLine" www.fastionline. org/docs//FOLDER-it-2009-159.pdf.

SORRENTINO et alii 2018

R. Sorrentino - E. Bortolini - F. Lugli - G. Mancuso - L. Buti - G. Oxilia et alii, Unravelling biocultural population structure in 4 th/3rd century BC Monterenzio Vecchio (Bologna, Italy) through a comparative analysis of strontium isotopes, non-metric dental evidence, and funerary practices, in "PLoS ONE" 13, 3 (2018), https://doi.org/10.1371/ journal.pone.0193796.

\section{SPAGNOLO GARZOLI 1999}

G. Spagnolo Garzoli (a cura di), Conubia gentium. La necropoli di Oleggio e la romanizzazione dei Vertamocori, Catalogo della mostra (Oleggio, 23 gennaio-30 aprile 1999), Torino 1999.

\section{SPAGNOLO GARZOLI 2009}

G. Spagnolo Garzoli (a cura di), I celti di Dormelletto, Gravellona Toce 2009.
STÖCKLI 1975

W.E. Stöckli, Chronologie der jüngeren Eisenzeit im Tessin, Basel 1975 (Antiqua, 2).

\section{SUTERMEISTER 1960}

G. Sutermeister, Sepolture del $2^{\circ}, 3^{\circ}$ sec. av. Cr. S. Lorenzo di Nerviano, in "Memorie" 18 (1960), pp. 9-11.

TASSINARI 2010

C. Tassinari, I materiali di scavo della casa etrusca di via A. Costa a Bologna, in M. Bentz - C. Reusser (Hrsgg.), Etruskisch-italische und römischrepublikanische Häuser, Atti del Colloquio (Bonn, 23-25 gennaio 2009), Bonn 2010, pp. 89-103.

\section{TIZZONI 1981}

M. Tizzoni, La cultura La Tène in Lombardia, in "Studi archeologici" 1 (1981), pp. 3-40.

Tizzoni 1981

M. Tizzoni, La tarda età del ferro nella Valle del Chiese, in "Annali del Museo. Notiziario di preistoria e archeologia. Civico Museo Gruppo Grotte Gavardo” 15 (1982), pp. 211-219.

\section{TizZONI 1984}

M. Tizzoni, I materiali della tarda età nelle Civiche Raccolte Archeologiche di Milano, "Notizie dal Chiostro del Monastero Maggiore", suppl. III, Milano 1984.

\section{TIZZONI 1985}

M. Tizzoni, I materiali della tarda età del Ferro al Museo Civico di Brescia, "Studi archeologici" 4 (1984).

THÉR - MANGEL 2021

R. Thér - T. Mangel 2021, Two trajectories of the development of pottery forming methods in central Europe in the Iron Age: The contribution of analysis of the orientation of components of a ceramic body, in "Journal of Archaeological Science: Reports" 35 (2021), pp. 1-12.

TORI et alii 2010

L. Tori - E. Carlevaro - Ph. Della Casa - L. Pernet - B. Schmid-Sikimić, La necropoli di Giubiasco (TI), vol. III. Le tombe dell'età del Bronzo, 
della prima età del Ferro e del La Tène antico e medio. La sintesi, Zürich 2010.

TRuCCO 1979

F. Trucco 1979, Aspetti e problemi dell'età di La Tène antica e media fra Ticino e Sesia, in "Notizie del Chiostro del Monastero Maggiore" XXIIIXXIV (1979), pp. 3-68.

\section{VAN OYEN 2017}

A. Van Oyen, Material Culture in the Romanization debate, in A. Lichtenberger - R. Raja (eds), The Diversity of Classical Archaeology, Turnhout 2017, pp. 287-300.

\section{VANNACCI LUNAZZI 1977}

G. Vannacci Lunazzi, Le necropoli preromane di Remedello Sotto e Ca' di Marco di Fiesse, Reggio Emilia 1977.

VERSLUYS 2014

M.J. Versluys, Understanding objects in motion. An archaeological dialogue on Romanization, in "Archaeological Dialogues" 21.1 (2014), pp. 120.

VITALI 2014a

D. Vitali, La vaisselle céramique 'celtique' des Bö̈ens cisalpins (IVe-IIIe s. av. J.-C.). Quelques considérations générales, in "Archaeologia Mosellana" 9 (2014), pp. 295-314.

VITALI 2014b

D. Vitali, I Celti d'Italia (IV-I secolo a.C.) tra identità $e$ assimilazioni, in C.C. LambergKarlovsky - B. Genito - B. Cerasetti (a cura di), 'My Life is like the Summer Rose' Maurizio Tosi e l'Archeologia come modo di vivere, Papers in honour of Maurizio Tosi for his 70th birthday, Oxford 2014, pp. $733-750$.

VOLONTÈ 1996

A.M. Volontè, Le ricerche e le scoperte a Parabiago prima degli anni '90, in Antichi silenzi 1996, pp. 23-39.

ZAMBONI 2016

L. Zamboni, Spina città liquida. Gli scavi 19771981 nell'abitato e $i$ materiali tardo-arcaici e classici,
Rahden 2016 (Zürcher Archäologische Forschungen, 3).

ZAMBONI 2018

L. Zamboni, Sepolture arcaiche della pianura emiliana. Il riconoscimento di una società di frontiera, Roma 2018 (Reditus, 1). 